\title{
Three-Dimensional Synaptic Organization of Layer III of the Human Temporal Neocortex
}

\author{
Nicolás Cano-Astorga ${ }^{1,2}$, Javier DeFelipe ${ }^{1,2,3}$ and Lidia Alonso-Nanclares ${ }^{1,2,3}$ \\ ${ }^{1}$ Laboratorio Cajal de Circuitos Corticales, Centro de Tecnología Biomédica, Universidad Politécnica de Madrid, \\ Madrid 28223, Spain, ${ }^{2}$ Instituto Cajal, Consejo Superior de Investigaciones Científicas (CSIC), Madrid 28002, \\ Spain and ${ }^{3}$ Centro de Investigación Biomédica en Red sobre Enfermedades Neurodegenerativas (CIBERNED), \\ ISCIII, Madrid 28031, Spain
}

Address correspondence to Lidia Alonso-Nanclares. Email: aidil@cajal.csic.es.

\begin{abstract}
In the present study, we have used focused ion beam/scanning electron microscopy (FIB/SEM) to perform a study of the synaptic organization of layer III of Brodmann's area 21 in human tissue samples obtained from autopsies and biopsies. We analyzed the synaptic density, 3D spatial distribution, and type (asymmetric/symmetric), as well as the size and shape of each synaptic junction of 4945 synapses that were fully reconstructed in 3D. Significant differences in the mean synaptic density between autopsy and biopsy samples were found ( 0.49 and 0.66 synapses $/ \mathrm{\mu m}^{3}$, respectively). However, in both types of samples (autopsy and biopsy), the asymmetric:symmetric ratio was similar (93:7) and most asymmetric synapses were established on dendritic spines (75\%), while most symmetric synapses were established on dendritic shafts (85\%). We also compared several electron microscopy methods and analysis tools to estimate the synaptic density in the same brain tissue. We have shown that FIB/SEM is much more reliable and robust than the majority of the other commonly used EM techniques. The present work constitutes a detailed description of the synaptic organization of cortical layer III. Further studies on the rest of the cortical layers are necessary to better understand the functional organization of this temporal cortical region.
\end{abstract}

Key words: autopsy, biopsy, cerebral cortex, electron microscopy, FIB-SEM

\section{Introduction}

Several electron microscope (EM) studies have been performed to estimate the density and types of synapses in the human temporal cortex with a range of different standard EM techniques (Davies et al. 1987; Huttenlocher and Dabholkar 1997; Tang et al. 2001; Alonso-Nanclares et al. 2008; Yakoubi et al. 2018, 2019). However, these estimations are usually applied to a relatively small number of EM sections, which increases statistical variability and affects the reliability of the results (reviewed in Merchán-Pérez et al. 2009). Furthermore, in these studies, many synapses cannot be properly classified as asymmetric or symmetric, and they are tagged as "uncharacterized" (DeFelipe et al. 1999). These missing data are critical at the circuit level, since higher or lower proportions of asymmetric and symmetric synapses are linked to differences in the excitatory/inhibitory balance of the cortical circuits (for reviews, see DeFelipe 2015; Froemke 2015; Zhou and Yu 2018; Sohal and Rubenstein 2019).

EM with serial section reconstruction is the gold standard method for studying the synaptic organization. However, it is rather time-consuming and challenging to obtain long series of sections. This is why reconstruction of large tissue volumes is usually not feasible. The introduction of automated or semiautomated EM techniques at the turn of this century represents a major advance in the study of synaptic organization, as long 
Table 1 Clinical information of the analyzed cases. Causes of death are shown in parentheses in the autopsy cases

\begin{tabular}{|c|c|c|c|c|}
\hline Case & Sex & Age (years) & Tissue type & Postmortem delay (h) \\
\hline AB1 & Male & 45 & $\begin{array}{l}\text { Autopsy } \\
\text { (cardiorespiratory arrest) }\end{array}$ & $<1$ \\
\hline $\mathrm{AB} 2$ & Female & 53 & $\begin{array}{l}\text { Autopsy } \\
\text { (pulmonary septic shock) }\end{array}$ & 4 \\
\hline AB3 & Male & 53 & $\begin{array}{l}\text { Autopsy } \\
\text { (bladder metastatic carcinoma) }\end{array}$ & 3.5 \\
\hline H38 & Male & 24 & Biopsy TLE & 0 \\
\hline $\mathrm{H} 18$ & Female & 31 & Biopsy TLE & 0 \\
\hline $\mathrm{H} 60$ & Male & 36 & Biopsy TLE & 0 \\
\hline $\mathrm{H} 47$ & Female & 41 & Biopsy TLE & 0 \\
\hline H94 & Male & 27 & Biopsy TLE & 0 \\
\hline
\end{tabular}

Note: TLE: temporal lobe epilepsy

series of consecutive sections can now be obtained with little user intervention (e.g., Denk and Horstmann 2004; Knott et al. 2008; Smith 2008; Merchán-Pérez et al. 2009; Kleinfeld et al. 2011; Helmstaedter et al. 2013; Kasthuri et al. 2015; Kubota et al. 2018).

Regarding the way in which human brain tissue is acquired, there are two main sources: 1) postoperative brain tissue (biopsy) obtained from patients suffering pharmacoresistant temporal lobe epilepsy or from patients with brain tumors and 2) that obtained from the autopsy of individuals with no recorded neurological or psychiatric alterations.

The advantage of using biopsies is that the resected tissue can be immediately immersed in the fixative. This is why the quality of the immunocytochemical staining at both the light and electron microscopy levels in human biopsy material has been shown to be comparable to that obtained in experimental animals (e.g., del Río and DeFelipe 1994). The problem is that, although brain tissue obtained at biopsy may come from part of a region that is considered "normal" from a pathological and physiological point of view, it is obtained from patients who did have an underlying brain-related disorder. Thus, autopsy may be the sole source of strictly normal tissue. The problem is that there can be a long delay between death and tissue collection (often over $5 \mathrm{~h}$ ), and the ultrastructure of the postmortem brain tissue is generally not well preserved, which makes the tissue unsuitable for detailed quantitative analysis. This is one of the main reasons why synaptic circuitry data for the normal human brain is so lacking. However, we have shown that the 3D reconstruction method using focused ion beam/scanning electron microscopy (FIB/SEM) can be applied to study the synaptic organization of the human brain obtained at autopsy in great detail, yielding excellent results (Domínguez-Álvaro et al. 2018, 2019, 2021a, 2021b; Montero-Crespo et al. 2020).

The main objective of this study is to analyze the synaptic organization of the neuropil of layer III of the anterior part of the middle temporal convolution (Brodmann's area 21; BA21; see Zilles and Amunts 2010) obtained from both biopsies and autopsies. It has been proposed that this cortical layer is critically involved in the emergence of cognitive functions, such as language (Thomson and Lamy 2007; Xu et al. 2016; Cheng et al. 2017) and most physiological studies in the human neocortex are performed in layers II and III (e.g., see Eyal et al. 2018; Gidon et al. 2020, and references therein). Thus, a detailed microanatomical analysis could be useful to better understand this cortical region by integrating morphological and physiological studies. We used FIB/SEM in normal brain tissue obtained at autopsy with a short postmortem delay (less than $4 \mathrm{~h}$ ) and from "normal" tissue obtained at biopsy (from patients with mesial temporal lobe epilepsy, with no fixation delay) to determine the synaptic organization in this particular brain region.

The present study also allows the comparison of the data from autopsy and biopsy using the same technique (FIB/SEM). Moreover, since this layer has been examined in previous studies using standard EM techniques and stereological methods (Alonso-Nanclares et al. 2008), the present study also aimed to compare standard EM techniques with the FIB/SEM to study the synaptic organization. For this purpose, we studied 4945 synapses, which were fully reconstructed in 3D. In particular, we analyzed the synaptic density and 3D spatial distribution, type of synapses (asymmetric/symmetric), as well as the shape and size of each synaptic junction. In addition, it was possible to determine the postsynaptic targets of 2304 axon terminals. Thus, data from the present work constitute a detailed description of the synaptic organization in layer IIIA of the human BA21, which may contribute to better understanding the synaptic organization of this layer.

\section{Materials and Methods}

\section{Tissue Preparation}

Human brain tissue was obtained from three autopsies and five biopsies (Table 1). The autopsy samples (with short postmortem delays of less than $4 \mathrm{~h}$ ) were obtained from two men and one woman (whose ages ranged from 45 to 53 years old) with no recorded neurological or psychiatric alterations (supplied by Unidad Asociada Neuromax, Laboratorio de Neuroanatomía Humana, Facultad de Medicina, Universidad de Castilla-La Mancha, Albacete). The sampling procedure was approved by the Institutional Ethical Committee. This human brain tissue has been used in previous EM studies (Domínguez-Álvaro et al. 2018, 2019, 2021a; Montero-Crespo et al. 2020). Biopsy samples were obtained during neurosurgery of epileptic patients suffering pharmaco-resistant mesial temporal lobe epilepsy in the Hospital de la Princesa (Madrid). Intraoperative electrocorticographic recordings revealed that the lateral neocortex of all these patients displayed normal activity. That is, no spikes, sharp waves, or slow activities were observed during intraoperative electrocorticography. A portion of the anterior part of the left temporal lobe was removed. In each 


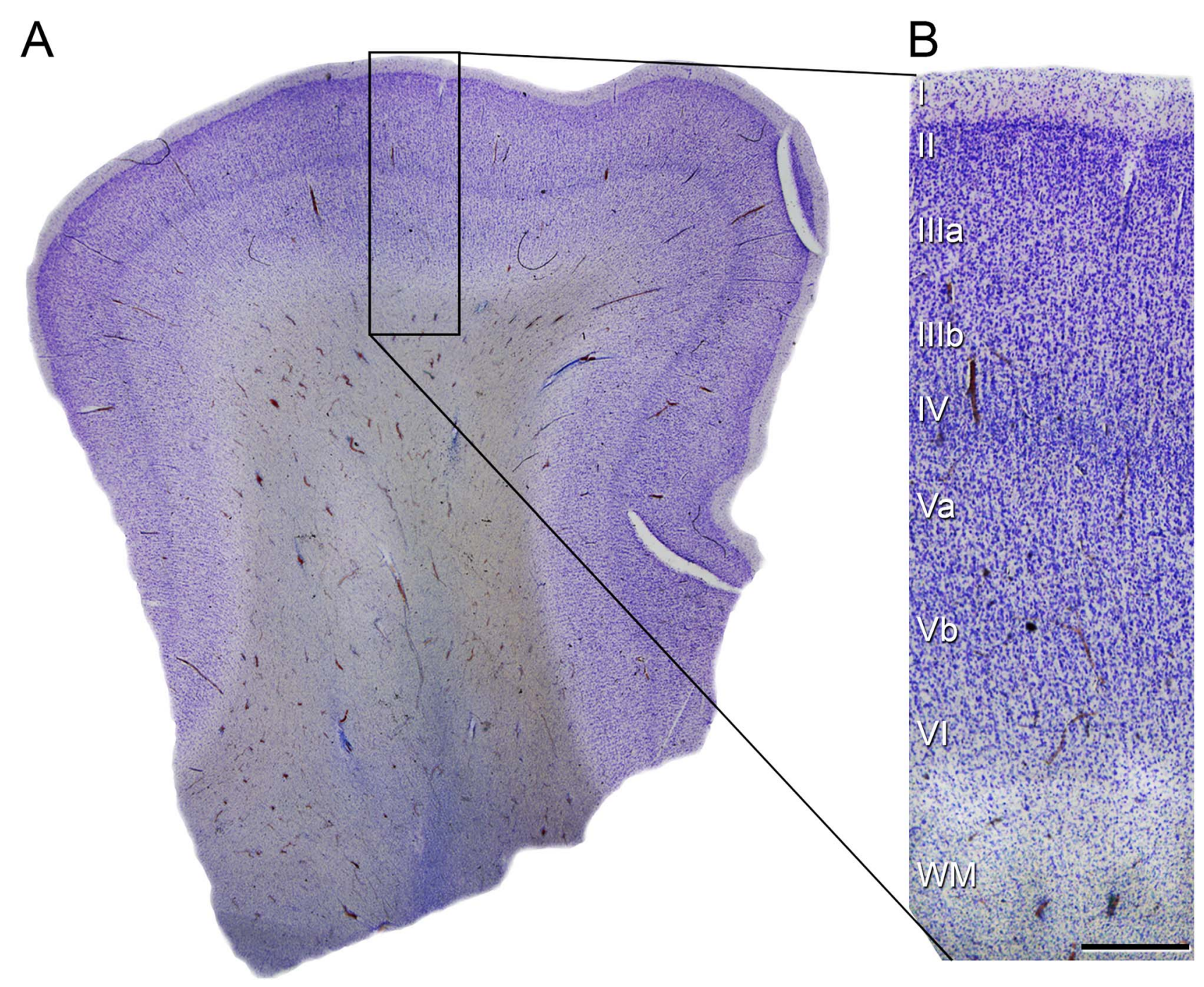

Figure 1. Coronal vibratome section of the human temporal neocortex. (A) Low-power photograph of a $100 \mu \mathrm{m}$-thick Nissl-stained section of temporal neocortex including Brodmann's area 21 (BA21). (B) Higher magnification of the boxed area in A, showing the laminar pattern (layers I to VI are indicated). WM: white matter. Scale bar shown in B indicates $1900 \mu \mathrm{m}$ in $\mathrm{A}$ and $400 \mu \mathrm{m}$ in $B$.

case, the patient's consent was obtained in accordance with the Helsinki Declaration, and all protocols were approved by the ethical committee at the Hospital de la Princesa (Madrid). This biopsy tissue has also been used in previous studies (Alonso-Nanclares et al. 2008).

After extraction, brain tissue was fixed in cold $4 \%$ paraformaldehyde (Sigma-Aldrich, St Louis, MO, USA) in $0.1 \mathrm{M}$ sodium phosphate buffer (PB; Panreac, 131965, Spain), pH 7.4 for 24$48 \mathrm{~h}$. After fixation, the tissue was washed in PB and sectioned coronally in a vibratome (150 $\mu \mathrm{m}$ thickness; Vibratome Sectioning System, VT1200S Vibratome, Leica Biosystems, Germany). Sections containing BA21 were selected and processed for Nissl staining to determine cytoarchitecture (Fig. 1).

\section{Electron Microscopy}

Sections containing BA21 were selected and postfixed for $24 \mathrm{~h}$ in a solution containing $2 \%$ paraformaldehyde, $2.5 \%$ glutaraldehyde (TAAB, G002, UK), and 0.003\% $\mathrm{CaCl}_{2}$ (Sigma, C-2661-500G, Germany) in sodium cacodylate (Sigma, C0250-500G, Germany) buffer $(0.1 \mathrm{M})$. The sections were treated with $1 \% \mathrm{OsO}_{4}$ (Sigma, 05500, Germany), 0.1\% potassium ferrocyanide (Probus, 23345, Spain), and $0.003 \% \mathrm{CaCl}_{2}$ in sodium cacodylate buffer $(0.1 \mathrm{M})$ for $1 \mathrm{~h}$ at room temperature. They were then stained with $1 \%$ uranyl acetate (EMS, 8473, USA), dehydrated, and flat-embedded in Araldite (TAAB, E021, UK) for $48 \mathrm{~h}$ at $60^{\circ} \mathrm{C}$ (DeFelipe and Fairén 1993). The embedded sections were then glued onto a blank Araldite block. Semithin sections (1-2 $\mu \mathrm{m}$ thick) were obtained from the surface of the block and stained with $1 \%$ toluidine blue (Merck, 115930, Germany) in 1\% sodium borate (Panreac, 141644, Spain). The last semithin section (which corresponds to the section immediately adjacent to the block surface) was examined under light microscope and photographed to accurately locate the neuropil regions to be examined (Fig. 2).

\section{Three-Dimensional Electron Microscopy}

The 3D study of the samples was carried out using a dual beam microscope (Crossbeam ${ }^{\circledR} 540$ electron microscope, Carl Zeiss NTS GmbH, Oberkochen, Germany). This instrument combines 


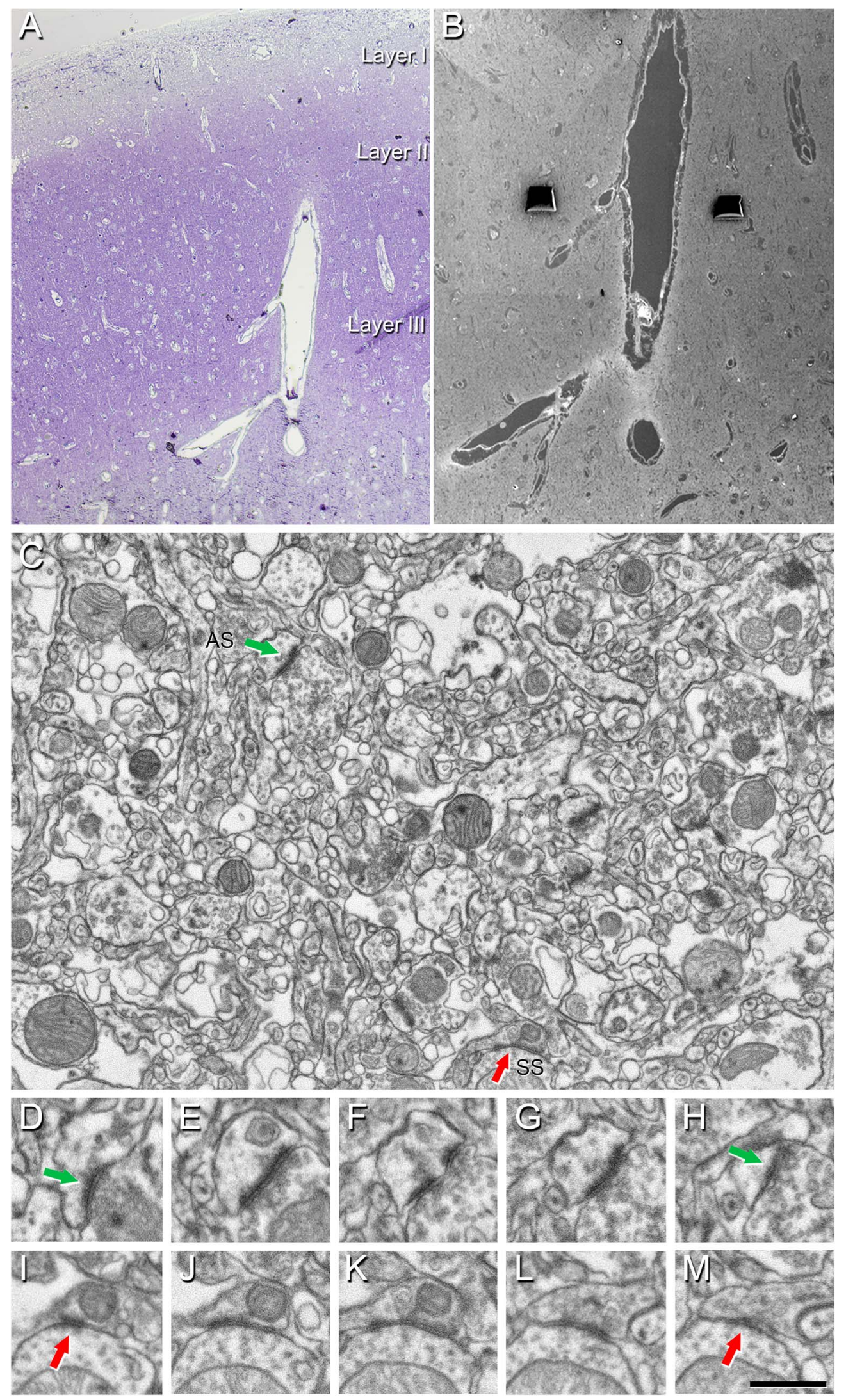

Figure 2. Correlative light/electron microscopy analysis of layer III of Brodmann's area 21 (BA21). (A) Delimitation of layers is based on the staining pattern of $1 \mu \mathrm{m}$-thick semithin section, stained with toluidine blue, which is adjacent to the block for FIB/SEM imaging (B). (B) SEM image showing a higher magnification of the blood vessels in A to illustrate the tissue surface with the trenches made for the neuropil analyses. (C) Serial image obtained by FIB/SEM showing the neuropil of an autopsy case (AB3); two synapses are indicated as examples of asymmetric (AS, green arrow) and symmetric synapses (SS, red arrow). Synapse classification was based on the examination of the full sequence of serial images; an AS can be visualized in $\mathrm{D}-\mathrm{H}$, and an SS in I-M. See Three-dimensional analysis of synapses for further details. Scale bar shown in M indicates $160 \mu \mathrm{m}$ in A, $100 \mu \mathrm{m}$ in B, $950 \mathrm{~nm}$ in C, and $550 \mathrm{~nm}$ in D-M. 
a high-resolution field-emission SEM column with a focused gallium ion beam (FIB), which permits removal of thin layers of material from the sample surface on a nanometer scale. As soon as one layer of material ( $20 \mathrm{~nm}$ thick) is removed by the FIB, the exposed surface of the sample is imaged by the SEM using the backscattered electron detector. The sequential automated use of FIB milling and SEM imaging allowed us to obtain long series of photographs of a 3D sample of selected regions (Merchán-Pérez et al. 2009). Image resolution in the xy plane was $5 \mathrm{~nm}$ /pixel. Resolution in the z-axis (section thickness) was $20 \mathrm{~nm}$, and image size was $2048 \times 1536$ pixels. These parameters were to obtain a large enough field of view where synaptic junctions could be clearly identified, in a reasonable amount of time (approximately $12 \mathrm{~h}$ per stack of images).

All measurements were corrected for tissue shrinkage that occurs during processing of sections (Merchán-Pérez et al. 2009). To estimate the shrinkage in our samples, we photographed and measured the area of the vibratome sections with ImageJ (ImageJ 1.51; NIH, USA), both before and after processing for electron microscopy. The section area values after processing were divided by the values before processing to obtain the volume, area, and linear shrinkage factors (Oorschot et al. 1991), yielding correction factors of 0.90, 0.93, and 0.97, respectively. Nevertheless, in order to compare with previous studies-in which either no correction factors had been included or such factors were estimated using other methods-in the present study, we provided both sets of data. Additionally, a correction in the volume of the stack of images for the presence of fixation artifact (i.e., swollen neuronal or glial processes) was applied after quantification with Cavalieri principle (Gundersen et al. 1988b; see Montero-Crespo et al. 2020). Every FIB/SEM stack was examined, and the volume artifact ranged from 0 to $9.4 \%$ of the volume stacks.

\section{Three-Dimensional Analysis of Synapses}

Stacks of images obtained by the FIB/SEM were analyzed using EspINA software (EspINA Interactive Neuron Analyzer, 2.1.9; https://cajalbbp.es/espina/ Fig. 3). As previously discussed (Merchán-Pérez et al. 2009), there is a consensus for classifying cortical synapses into asymmetric synapses (AS; or type I) and symmetric synapses (SS; or type II). The main characteristic distinguishing these synapses is the prominent or thin postsynaptic density, respectively (Fig. 2). Also, these two types of synapses correlate with different functions: AS are mostly glutamatergic and excitatory, while SS are mostly GABAergic and inhibitory (DeFelipe and Fariñas 1992). Nevertheless, in single sections, the synaptic cleft and the pre- and postsynaptic densities are often blurred if the plane of the section does not pass at right angles to the synaptic junction. Since the software EspINA allows navigation through the stack of images, it was possible to unambiguously identify every synapse as AS or SS based on the thickness of the postsynaptic density (PSD) (Merchán-Pérez et al. 2009). Synapses with prominent PSDs are classified as AS, while thin PSDs are classified as SS (Gray 1959; Peters et al. 1991; Fig. 2). In addition, geometrical features—such as size and shape-and spatial distribution features (centroids) of each reconstructed synapse were also calculated by EspINA.

This software also extracts the Synaptic Apposition Area (SAS) and provides its morphological measurements (Fig. 3). Given that the pre- and postsynaptic densities are located face to face, their surface areas are comparable (for details, see Morales et al. 2013). Since the SAS comprises both the active zone and the
PSD, it is a functionally relevant measure of the size of a synapse (Morales et al. 2013).

EspINA was also used to visualize each of the reconstructed synapses in $3 \mathrm{D}$ and to detect the possible presence of perforations or deep indentations in their perimeters. Regarding the shape of the PSD, the synaptic junctions could be classified into four main categories, according to the categories proposed by Santuy et al. (2018a): macular (disk-shaped PSD), perforated (with one or more holes in the PSD), horseshoe-shaped (with an indentation), and fragmented (disk-shaped PSDs with no connection between them) (Fig. 4A).

To identify the postsynaptic targets of the synapses, we navigated through the image stack using EspINA to determine whether the postsynaptic element was a dendritic spine (spine, for simplicity) or a dendritic shaft. As previously described in Domínguez-Álvaro et al. (2021a, 2021b), unambiguous identification of spines requires the spine to be visually traced to the parent dendrite (Figs 5 and 6; Movies 1 and 2). We refer to them as fully reconstructed spines. Additionally, when synapses were established on a spine head-shaped postsynaptic element whose neck could not be followed to the parent dendrite, we identified these elements as nonfully reconstructed spines. These nonfully reconstructed spines were identified on the basis of their size and shape, the lack of mitochondria, and the presence of a spine apparatus-or because they were filled with a characteristic fluffy material (used to describe the fine and indistinct filaments present in the spines) - a term coined by Peters et al. (1991) (see also del Río and DeFelipe 1995). For simplicity, we will refer to the fully reconstructed and nonfully reconstructed spines as spines, unless otherwise specified. We also recorded the presence of single or multiple synapses on a single spine. Furthermore, we determined whether the target dendrite had spines or not.

\section{Quantification of the Synaptic Density}

\section{Direct Counting Method}

EspINA provided the 3D reconstruction of every synapse and allowed the application of an unbiased 3D counting frame (CF). This CF is a regular rectangular prism enclosed by three acceptance planes and three exclusion planes marking its boundaries (Fig. 3). All objects within the CF are counted, as are those intersecting any of the acceptance planes, while objects that are outside the $\mathrm{CF}$, or intersecting any of the exclusion planes, are not counted. Thus, the number of synapses per unit volume was calculated directly by dividing the total number of synapses counted by the volume of the CF (Merchán-Pérez et al. 2009). This method was used in all 22 stacks of images.

\section{Disector Method}

In addition to counting directly, we also estimated the synaptic density in three stacks of images from one biopsy case (H47) using the disector method, which is based on counting the number of profiles $\left(\Sigma Q_{-}\right)$that are present in a given section (the reference section) and that disappear in another section (the look-up section), located at a known distance in the $z$ axis within the unbiased CF (Gundersen 1977). The number of synapses per unit volume (NV) is calculated using the formula $\mathrm{NV}=\Sigma \mathrm{Q}-/ \mathrm{ah}$, where "a" is the area of the unbiased CF and " $\mathrm{h}$ " is the distance between the two sections. Usually, after one disector is calculated, the top and bottom microphotographs are swapped and used as the new reference and look-up sections. Thus, any given pair of sections yields two estimates (Gundersen 

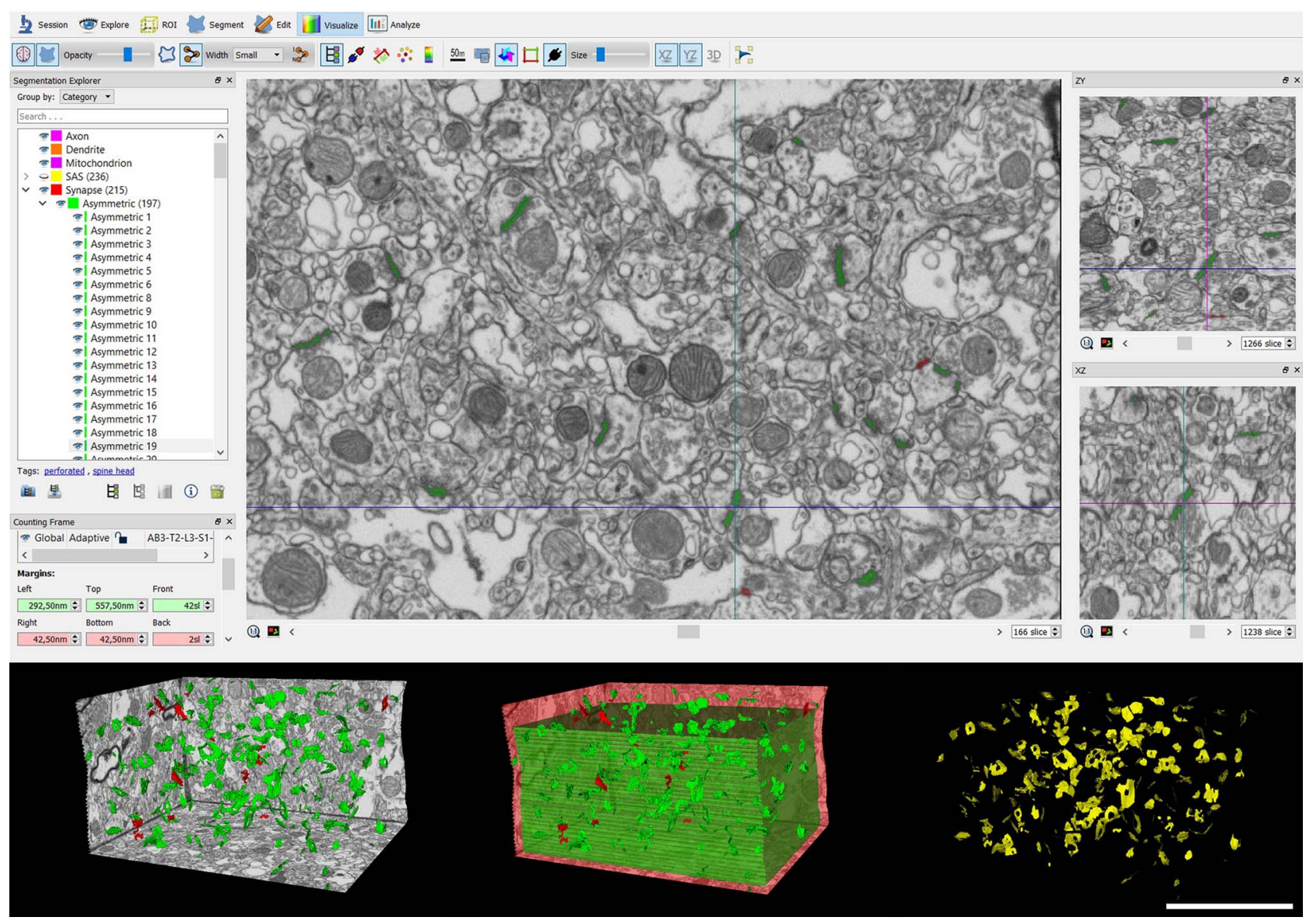

Figure 3. Screenshot of the EspINA software user interface. (Top) In the main window, the sections are viewed through the xy plane (as obtained by FIB/SEM microscopy). The other two orthogonal planes, $y z$ and $x z$, are also shown in adjacent windows (on the right). (Bottom left) The 3D windows show the three orthogonal planes and the 3D reconstruction of AS (green) and SS (red) segmented synapses. (Bottom center) The counting frame composed by three exclusion planes (light red) and three inclusion planes (light green) with the reconstructed synapses. (Bottom right) The computed SAS for each reconstructed synapse (in yellow). Scale bar shown in the bottom right indicates $5 \mu \mathrm{m}$ for all three of the compositions shown at the bottom.

et al. 1988a). However, not all disectors are useful to estimate the number of synapses, since the distance $h$ should not exceed $1 / 4$ to $1 / 3$ of the mean particle length (Gundersen et al. 1988a). Given that the mean cross-sectional length of synaptic profiles is between 280 and $350 \mathrm{~nm}$ (DeFelipe et al. 1999), the h value of the disectors should not exceed $70 \mathrm{~nm}$ if the most conservative approach is taken (1/4 of 280). In practice, we chose three times the mean section thickness $(60 \mathrm{~nm})$ and an unbiased CF that represent an area of $60.69 \mu^{2}$. The variability of data obtained by the disector method tends to reduce as the number of sections sampled increases (Merchán-Pérez et al. 2009); thus, we established at least 101 pairs of disectors as a suitable value to perform the quantification.

\section{Spatial Distribution Analysis of Synapses}

To analyze the spatial distribution of synapses, spatial point pattern analysis was performed as described elsewhere (Antón-Sánchez et al. 2014; Merchán-Pérez et al. 2014). Briefly, we compared the actual position of centroids of synapses with the complete spatial randomness (CSR) model-a random spatial distribution model that defines a situation where a point is equally likely to occur at any location within a given volume. For each of the 22 FIB/SEM stacks of images, we calculated three functions commonly used for spatial point pattern analysis:
G, F, and $\mathrm{K}$ functions. As described in Merchán-Pérez et al. (2014) (see also Antón-Sánchez et al. 2014), the G function, also called the nearest-neighbor distance cumulative distribution function or the event-to-event distribution, is-for a distance $d$-the probability that a typical point separates from its nearest neighbor by a distance of $d$ at the most. The F function, also known as the empty space function or the point-to-event distribution, is-for a distance $d$-the probability that the distance of each point (in a regularly spaced grid of $L$ points superimposed over the sample) to its nearest synapse centroid is $d$ at the most. The $\mathrm{K}$ function, also called the reduced second moment function or Ripley's function, is-for a distance $d$ - the expected number of points within a distance $d$ of a typical point of the process divided by the intensity $\lambda$. An estimation of the $\mathrm{K}$ function is given by the mean number of points within a sphere of increasing radius $d$ centered on each sample point, divided by an estimation of the expected number of points per unit volume. This study was carried out using the Spatstat package and R Project program (Baddeley et al. 2015).

\section{Statistical Analysis}

Statistical comparisons of synaptic density and the area of the SAS were carried out using the unpaired Mann-Whitney (MW) nonparametric U test (normality and homoscedasticity criteria 
A

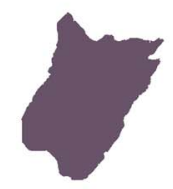

Macular

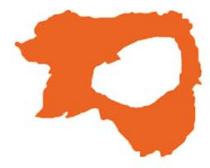

Perforated

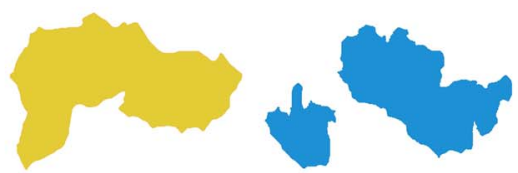

Horseshoe-shaped

Fragmented
C

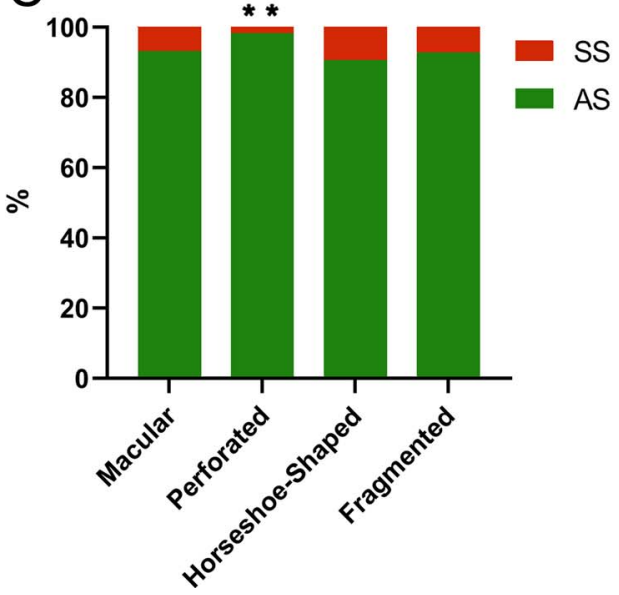

B

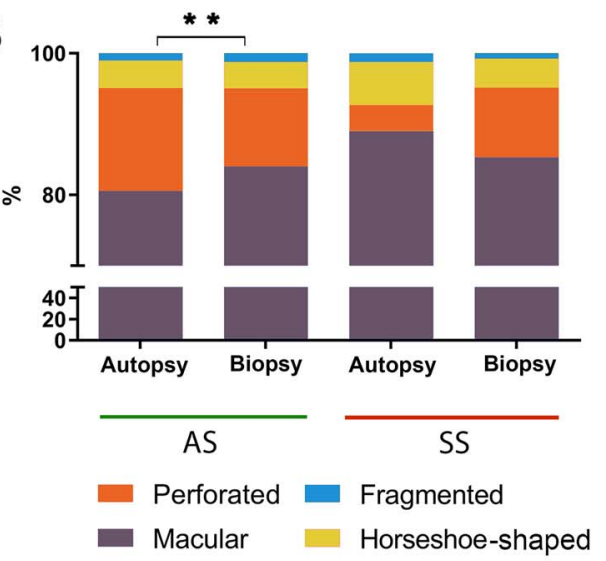

Figure 4. Analyses of the synaptic shape distribution in autopsy and biopsy samples from layer III of Brodmann's area 21 (BA21). (A) Schematic representation of the shape of the synaptic junctions: macular synapses with continuous disk-shaped PSD; perforated synapses with holes in the PSD; horseshoe-shaped synapses with tortuous horseshoe-shaped perimeter with an indentation; and fragmented synapses with two or more PSDs with no connections between them. (B) Proportion of macular, perforated, horseshoe-shaped, and fragmented AS and SS in autopsy and biopsy samples. In biopsy samples, macular synapses were significantly more frequent $\left(\chi^{2} ; P=0.0054\right)$, while in autopsy samples, perforated synapses were more frequent $\left(\chi^{2} ; P=0.0013\right)$. (C) Proportion of AS and SS belonging to each morphological category in autopsy samples. The perforated synapses were significantly more frequent among AS than SS ( $\left.\chi^{2} ; P=0.0028\right)$. (D) Proportion of AS and SS belonging to each morphological category in biopsy samples.

were not met). To identify possible differences regarding the area of the SAS related to its shape or its postsynaptic target, a Kruskal-Wallis (KW) nonparametric test was performed (normality and homoscedasticity criteria were not met). Frequency distribution analysis of the SAS was performed using Kolmogorov-Smirnov (KS) nonparametric test. To perform statistical comparisons of AS and SS proportions regarding their synaptic morphology and their postsynaptic target, chi-square $\left(\chi^{2}\right)$ test was used for contingency tables. The same method was used to compare between autopsies and biopsies in relation to the synaptic type, the shape of the synaptic junctions, and their postsynaptic target.

Statistical studies were performed with the GraphPad Prism statistical package (Prism 8.00 for Windows, GraphPad Software Inc., USA), Spatstat package and R Project program (Baddeley et al. 2015).

\section{Results}

The following results were obtained in the neuropil (i.e., excluding cell bodies, blood vessels, and major dendritic trunks).

\section{Synaptic Density}

Direct Counting Method

A total of 22 stacks of images of the BA21 layer IIIA neuropil (approximately 550-750 $\mu \mathrm{m}$ from pial surface) were obtained (three stacks per case, in six cases; and two stacks per case, in two cases; total volume studied: $11158{\mu \mathrm{m}^{3}}^{3}$ ) from both autopsy and biopsy samples. The number of sections per stack ranged from 259 to 317, which corresponds to a volume examined per stack ranging from 452 to $553{\mu \mathrm{m}^{3}}^{3}$ (mean: $507 \mu^{3}$ ). A total of 6595 synapses were individually identified and reconstructed in 3D; of these, 4945 synapses were analyzed after discarding incomplete synapses or those touching the exclusion edges of the CF.

A total of 1337 synapses were considered for analysis from autopsy samples (total volume studied: $3659 \mu^{3}$ ) and 3608 from biopsy samples (total volume studied: $7498 \mu^{3}$ ) (Table 2; Supplementary Table 1). The synaptic density values were obtained by dividing the total number of synapses included within the CF by its total volume. The mean synaptic density range was $0.44-0.54$ synapses/ $\mu^{3}$ from the autopsy samples and $0.58-0.77$ synapses $/ \mu^{3}$ in the case of the biopsy samples 


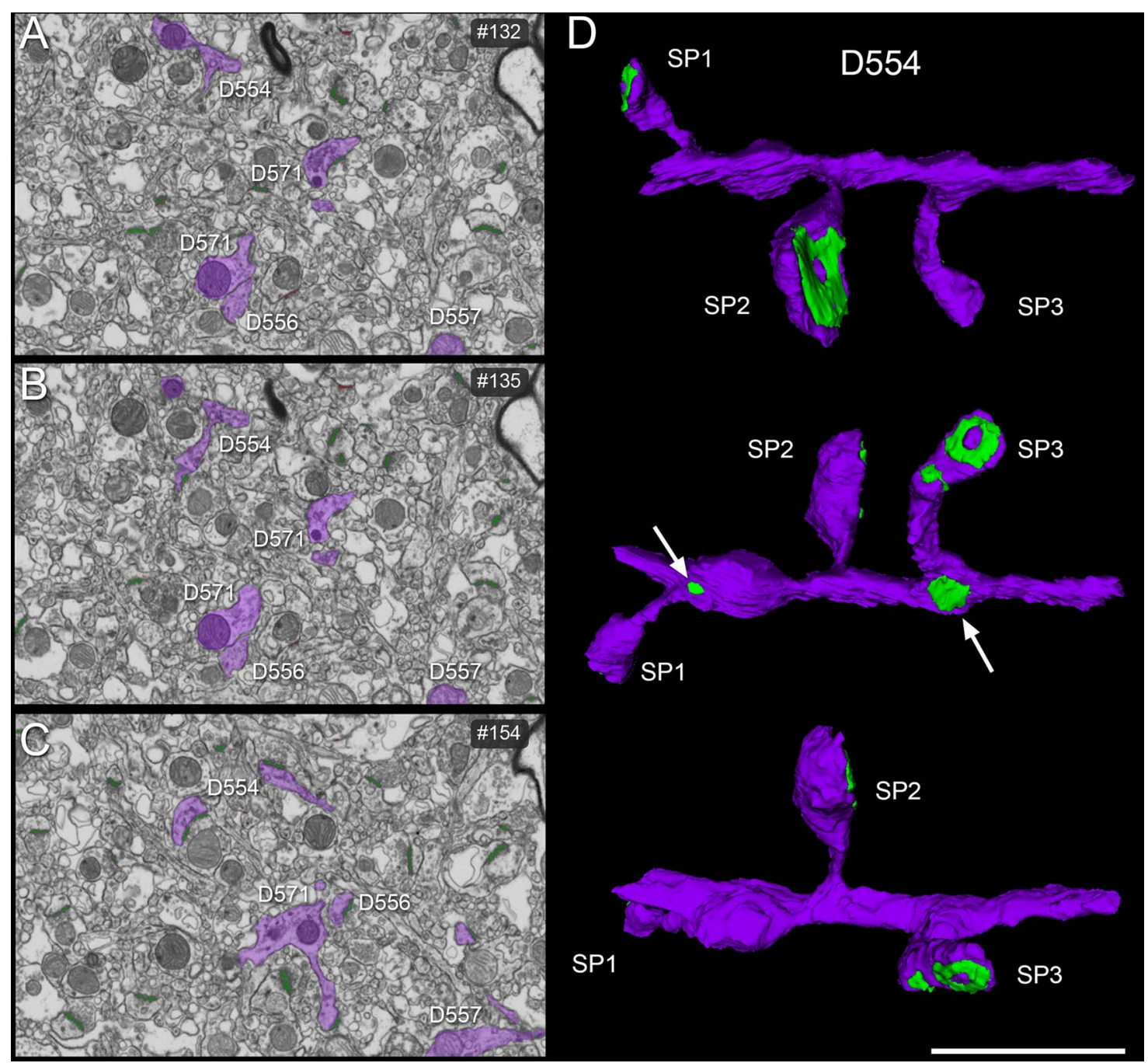

Figure 5. 3D reconstruction of dendritic segments from FIB/SEM serial images. (A-C) Composition of electron microscopy serial images showing dendritic segments (D554, D556, D557, and D571). Some reconstructions of these dendritic segments are displayed in D and Fig. 6. (D) 3D reconstructed dendritic segment D554 showing three views after rotation of the major dendritic axis at different angles. Three dendritic spines (SP) are shown, establishing asymmetric synapses (green) with different morphologies: perforated (SP2 and SP3) and macular (SP1). Arrows indicate two macular asymmetric synapses on the dendritic shaft. More examples of postsynaptic element characterization are shown in Supplementary Figures 1 and 2. Scale bar (in D) indicates $3.75 \mu \mathrm{m}$ in A-C and $2 \mu \mathrm{m}$ in D (see Movies 1 and 2).

(Table 2; Supplementary Table 1). The values obtained in biopsy samples were significantly higher (MW, $P=0.0357$ ) (Fig. 7).

\section{Disector Method}

Quantification of the synapses using the disector method was performed to evaluate possible differences between the two different quantification methods. The disector method was used to estimate the synaptic density in the same stacks that were analyzed with the direct counting method, as well as in an additional three stacks of images from the same case (biopsy H47). A total of 319, 410, and 434 synaptic profiles were counted ( $\Sigma \mathrm{Q}-$ ) in FIB/SEM stacks number 1, 2, and 3, respectively. Applying the formula $\mathrm{N}_{\mathrm{V}}=\Sigma \mathrm{Q}-/ \mathrm{ah}\left(\mathrm{N}_{\mathrm{V}}\right.$ : number of synapses per unit volume; a: area of the unbiased counting frame; $h$ : distance between the reference and the look-up sections), we obtained the following synaptic densities: 0.433 synapses $/ \mu \mathrm{m}^{3}$, 0.557 synapses $/ \mu \mathrm{m}^{3}$, and 0.590 synapses $/ \mu \mathrm{m}^{3}$ in the three stacks, respectively (Supplementary Fig. 3). These values were similar to the values obtained in the same stacks by the direct counting method (Supplementary Fig. 3). That is, we did not find any difference in the synaptic densities using different estimation methods, when applied to the same samples-providing that a relatively large number of disectors were used for the estimation (101 pairs of disectors).

\section{Proportion of Asymmetric/Symmetric Synapses}

The proportions of AS and SS were calculated in all samples. Since synapses were fully reconstructed in $3 \mathrm{D}$, it was possible to classify all synaptic contacts as AS and SS based on their PSDs (Fig. 2).

The proportion of AS:SS was 93:7 in both autopsy and biopsy samples (Table 2; Supplementary Table 1). Hence, no significant differences in proportions between autopsy samples and biopsy samples $\left(\chi^{2}, P>0.05\right)$ were observed.

The AS:SS ratios obtained by the disector method were: 92:8, 91:9, and 89:11, respectively, in the three stacks of images analyzed. Estimation of the AS:SS ratio using the direct counting method yielded similar results-93:7, 93:7, and 89:11, respectively. In summary, no marked differences between 


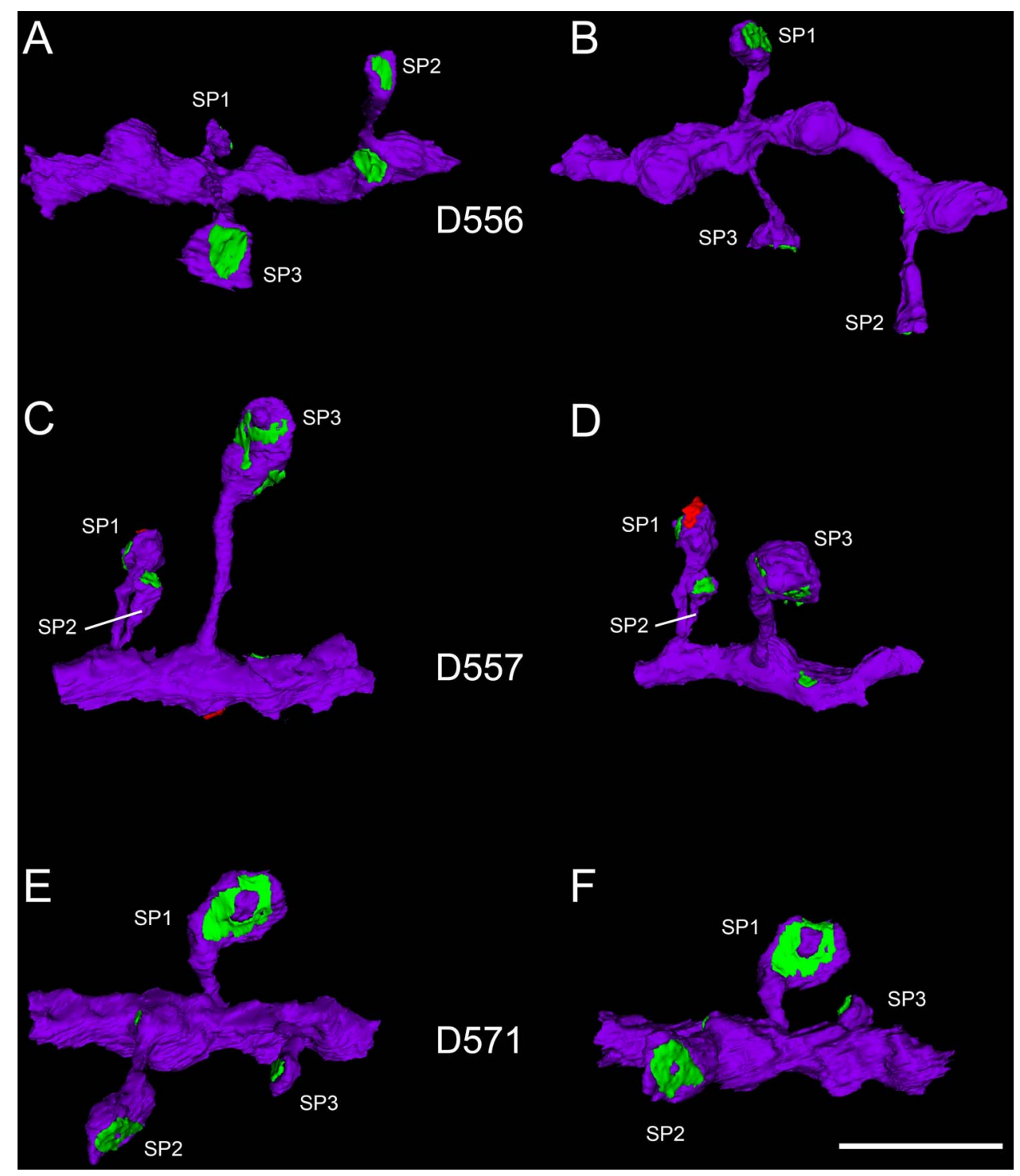

Figure 6. Examples of 3D reconstructed dendritic segments (purple) establishing asymmetric (green) and symmetric (red) synapses. A, B; C, $D$; and $E$, $F$ are pairs of views after axis rotation of the dendritic segments D556 (A, B), D557 (C, D), and D571 (E, F) at different angles. Note the different sizes and shapes of the synaptic junctions. Dendritic spines SP1 and SP3 of D557 established two synapses. More examples of postsynaptic element characterization are shown in Supplementary Figures 1 and 2. Scale bar (in F) indicates $2 \mu \mathrm{m}$ in A-F.

the AS:SS ratios of biopsy and autopsy samples were found, regardless of which one of the two methods was used.

\section{Three-Dimensional Spatial Synaptic Distribution}

To analyze the spatial distribution of the synapses, the actual position of each of the synapses in each stack of images was compared with a random spatial distribution model (CSR). For this, the functions $\mathrm{G}, \mathrm{K}$, and F were calculated in the 22 stacks of images analyzed. One stack of images did not fit into the CSR model and it showed a slight tendency to cluster. In the remaining samples, 21 out 22 stacks, the 3 spatial statistical functions resembled the theoretical curve that simulates the random spatial distribution pattern, which indicated that synapses fitted a random spatial distribution model, both in the autopsy samples and the biopsy samples (Supplementary Fig. 4).
In addition, the distance of each synapse to its nearest synapse was estimated. The mean distance to its nearest neighbor measured between centroids of synaptic junctions showed significant differences (MW; $P=0.0357$ ) between autopsy and biopsy samples (817 $\mathrm{nm}$ and $719 \mathrm{~nm}$, respectively; Table 2; Supplementary Table 1), indicating that, although the synapses fitted random spatial distribution in both groups, the synapses were more separated in the autopsy samples than in the biopsy samples.

\section{Study of the Synapse Characteristics}

\section{Synaptic Size}

The study of the synaptic size was carried out analyzing the area of the SAS of each synapse identified and 3D reconstructed in all FIB/SEM stacks (Fig. 3). 
Table 2 Accumulated data obtained from the ultrastructural analysis of neuropil from layer III of BA21 in samples obtained from autopsy and biopsy

\begin{tabular}{|c|c|c|c|c|c|c|c|c|c|c|}
\hline Group & No. AS & No. SS & $\begin{array}{l}\text { No. all } \\
\text { synapses }\end{array}$ & $\begin{array}{l}\% \text { AS } \\
(\text { mean } \pm S D)\end{array}$ & $\begin{array}{l}\% \mathrm{SS} \\
(\text { mean } \pm \mathrm{SD})\end{array}$ & $\begin{array}{l}\text { CF volume } \\
\left(\mu \mathrm{m}^{3}\right)\end{array}$ & $\begin{array}{l}\text { No. AS } \\
\text { synapses } / \mu m^{3} \\
(\text { mean } \pm \mathrm{SD})\end{array}$ & $\begin{array}{l}\text { No. SS } \\
\text { synapses/ } / \mu \mathrm{m}^{3} \\
(\text { mean } \pm \mathrm{SD})\end{array}$ & $\begin{array}{l}\text { No. } \\
\text { synapses } / \mu \mathrm{m}^{3} \\
(\text { mean } \pm \mathrm{SD})\end{array}$ & $\begin{array}{l}\text { Distance to } \\
\text { the nearest } \\
\text { neighbor } \\
(\mathrm{nm} \text {; } \\
\text { mean } \pm \mathrm{SD} \text { ) }\end{array}$ \\
\hline Autopsy & 1255 & 82 & 1337 & $93.45 \pm 2.15$ & $6.55 \pm 2.15$ & 2704 (2435) & $\begin{array}{l}0.46 \pm 0.06 \\
(0.51 \pm 0.06)\end{array}$ & $\begin{array}{l}0.03 \pm 0.01 \\
(0.03 \pm 0.01)\end{array}$ & $\begin{array}{l}0.49 \pm 0.05 \\
(0.55 \pm 0.05)\end{array}$ & $\begin{array}{l}817 \pm 51 \\
(793 \pm 49)\end{array}$ \\
\hline Biopsy & 3363 & 245 & 3608 & $93.26 \pm 1.36$ & $6.74 \pm 1.36$ & 5439 (4898) & $\begin{array}{l}0.62 \pm 0.07 \\
(0.69 \pm 0.08)\end{array}$ & $\begin{array}{l}0.05 \pm 0.01 \\
(0.05 \pm 0.01)\end{array}$ & $\begin{array}{l}0.66 \pm 0.07 \\
(0.74 \pm 0.08)\end{array}$ & $\begin{array}{l}719 \pm 17 \\
(700 \pm 17)\end{array}$ \\
\hline Total & 4618 & 327 & 4945 & $93.33 \pm 1.55$ & $6.67 \pm 1.55$ & 8143 (7333) & $\begin{array}{l}0.56 \pm 0.10 \\
(0.62 \pm 0.11)\end{array}$ & $\begin{array}{l}0.04 \pm 0.01 \\
(0.04 \pm 0.01)\end{array}$ & $\begin{array}{l}0.60 \pm 0.11 \\
(0.67 \pm 0.12)\end{array}$ & $\begin{array}{l}756 \pm 59 \\
(735 \pm 56)\end{array}$ \\
\hline
\end{tabular}

Notes: Data in parentheses have not been corrected for shrinkage. The data for individual cases are shown in Supplementary Table 1. AS: asymmetric synapses; CF: counting frame; SD: standard deviation; SS: symmetric synapses

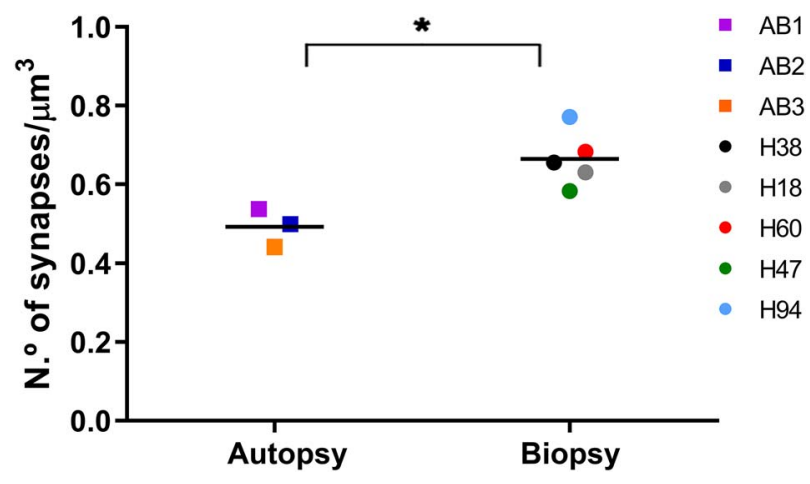

Figure 7. Mean synaptic density from autopsy and biopsy samples analyzed in layer III of Brodmann's area 21 (BA21). Each symbol represents a single case according to the colored key (squares for autopsy samples, circles for biopsy samples). Significant differences in the mean synaptic density between autopsy and biopsy samples were found (MW; $P=0.0357$ ).

In both autopsy and biopsy samples, the average size (measured by the area of the SAS) of the synapses showed significant differences (MW; $P<0.0002$ ) between AS and SS, with the latter being smaller than the former (Table 3; Supplementary Table 2). These differences were also found in the frequency distribution analyses in both the autopsy samples (KS; $P<0.0001)$ and the biopsy samples (KS; $P=0.0011$ ), again indicating that AS were larger than SS in both the autopsy (Fig. 8A) and biopsy (Fig. 8B) samples.

Upon analysis of the average area of the SAS of AS synapses in the autopsy and biopsy samples, no significant differences were found (MW; $P>0.05$ ), and the frequency distribution analyses did not reveal significant differences (KS; $P>0.05$; Fig. $8 C$ ). The number of SS examined in the autopsy samples was not sufficient to perform a robust statistical analysis.

\section{Synaptic Shape}

The synapses were classified into four categories: macular (with a flat, disk-shaped PSD), perforated (with one or more holes in the PSD), horseshoe (with an indentation in the perimeter of the PSD), or fragmented (with two or more physically discontinuous PSDs) (Fig. 4A; for a detailed description, see Santuy et al. 2018a; Domínguez-Álvaro et al. 2019).

In the autopsy samples, a total of 1255 AS were identified and $3 \mathrm{D}$ reconstructed. The vast majority $(80.6 \%)$ presented macular
Table 3 Area $\left(\mathrm{nm}^{2}\right)$ of the SAS from autopsy and biopsy samples of layer III of BA21

\begin{tabular}{|c|c|c|}
\hline Group & $\begin{array}{l}\text { Type of } \\
\text { synapse }\end{array}$ & Area of SAS $\left(\mathrm{nm}^{2}\right)$ \\
\hline \multirow[t]{2}{*}{ Autopsy } & AS & $111164 \pm 13750(103383 \pm 12787)$ \\
\hline & SS & $59683 \pm 13394(55505 \pm 12457)$ \\
\hline \multirow[t]{2}{*}{ Biopsy } & AS & $109690 \pm 6356(102011 \pm 5911)$ \\
\hline & SS & $81304 \pm 7878(75613 \pm 7327)$ \\
\hline \multirow[t]{2}{*}{ Total } & AS & $110243 \pm 5895(102526 \pm 5483)$ \\
\hline & SS & $73196 \pm 7553(68072 \pm 7024)$ \\
\hline
\end{tabular}

Notes: All data are expressed in $\mathrm{nm}^{2}$ as mean \pm sem. Data in parentheses have not been corrected for shrinkage. The data for individual cases are shown in Supplementary Table 2. AS: asymmetric synapses; SAS: synaptic apposition surface; sem: standard error of the mean; SS: symmetric synapses

morphology, followed by $14.5 \%$ perforated, $3.9 \%$ horseshoeshaped, and $1 \%$ fragmented (Table 4; Supplementary Table 3). For the SS, a total of 82 synapses were identified and reconstructed in 3D, and the majority also presented macular morphology (89\%). Of the rest, $3.7 \%$ were perforated, $6.1 \%$ were horseshoe-shaped, and $1.2 \%$ were fragmented (Table 4; Supplementary Table 3).

In the biopsy samples, a total of 3363 AS were identified and 3D reconstructed. Similarly, most of them (84\%) presented macular morphology, followed by $11 \%$ perforated, $3.8 \%$ horseshoeshaped, and 1.2\% fragmented (Table 4; Supplementary Table 3). For the SS, a total of 245 synapses were identified and reconstructed in 3D. The majority presented macular morphology $(85.3 \%)$. Of the rest, $9.8 \%$ were perforated, $4.1 \%$ were horseshoe-shaped, and $0.8 \%$ were fragmented (Table 4 ; Supplementary Table 3).

To determine whether the shape of the synapses was related to the sample type, the frequency distributions of the morphological synaptic categories, of both AS and SS, were analyzed. Regarding AS, significant differences between biopsy and autopsy samples were found in the frequency distribution of the macular $\left(\chi^{2}, P=0.0054\right)$ and the perforated synapses $\left(\chi^{2}\right.$, $P=0.0013)$, indicating that macular synapses were more frequent in the biopsy samples, while perforated synapses were more frequent in the autopsy samples (Fig. 4B). Concerning SS, the numbers of synapses examined separately in the autopsy and biopsy samples were not sufficient to perform a robust statistical analysis. 

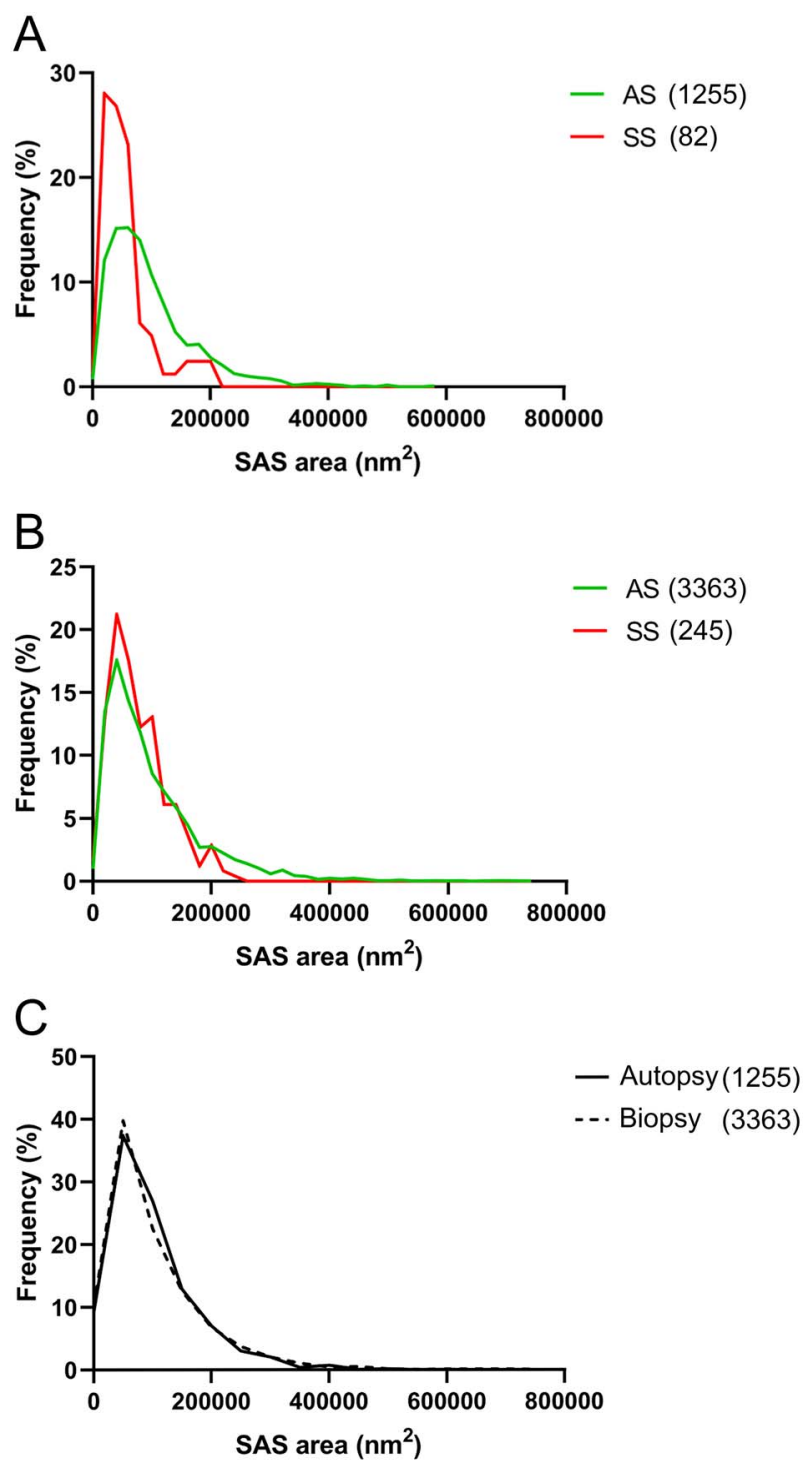

Figure 8. Frequency distribution of synaptic size (in terms of the SAS area). AS (green) were significantly larger than SS (red) in both autopsy samples (A; KS; $P<0.0001)$ and biopsy samples $(B ; K S ; P=0.0011)$. No significant differences were found in AS size between autopsy (continuous line) and biopsy (discontinuous line) samples ( $C ; \mathrm{KS} ; \mathrm{P}>0.05)$. Absolute numbers of analyzed synapses are shown in parentheses in the figure. AS: asymmetric synapses; SAS: synaptic apposition surface; SS: symmetric synapses.

Regarding synaptic type (AS or SS) in the autopsy samples, from the total macular synapses, $93.3 \%$ were AS and $6.7 \%$ were SS. This proportion was maintained $\left(\chi^{2}, P>0.05\right)$ in the case of horseshoe-shaped and fragmented synapses (91:9 and 93:7 of AS:SS, respectively), but changed significantly $\left(\chi^{2}, P=0.028\right)$ in the case of the perforated synapses, which were comprised of 98.4\% AS and 1.6\% SS (Fig. 4C). Thus, in the autopsy samples, the ratio of AS to SS was greater in the case of perforated synapses than it was for the other morphological categories.

Regarding synaptic type (AS or SS) in the biopsy samples, from the total macular synapses, $93.1 \%$ were AS and $6.9 \%$ were SS. This proportion was maintained $\left(\chi^{2}, P>0.05\right)$ in all morphological categories (perforated: 94:6; horseshoe-shaped: 93:7; fragmented: 95:5). Thus, in the biopsy samples, no one morphological category stood out from the others as having a greater frequency of a particular synaptic type (AS or SS) (Fig. 4D).

\section{Synaptic Size and Shape}

We also determined whether the shape of the synapses was related to their size. For this purpose, the areas of the SAS-of both AS and SS-were analyzed according to the synaptic shape. We found that the area of the macular AS was significantly smaller than the area of the perforated, horseshoe, and fragmented AS (KW, $P<0.0001)$ in both autopsy samples (Supplementary Fig. 5A) and biopsy samples (Supplementary Fig. 5B). Concerning SS, the number of synapses examined separately in the autopsy and biopsy samples was not sufficient to perform a robust statistical analysis.

\section{Study of the Postsynaptic Elements}

Postsynaptic targets were identified and classified as dendritic spines (axospinous synapses) or dendritic shafts (axodendritic synapses). We also distinguished whether the synapse was located on the neck or head of the spine (Fig. 5). When the postsynaptic element was identified as a dendritic shaft, it was classified as "with spines" or "without spines (Table 5).

In the autopsy samples, the postsynaptic elements of 1028 synapses were determined; of these, $63.62 \%$ were AS established on spine heads, $28.40 \%$ were AS on dendritic shafts, $5.44 \%$ were SS on dendritic shafts, and $1.28 \%$ were AS on spine necks (Fig. 9A). The two least frequent types of synapses were SS established on spine heads (1.07\%) and SS on spine necks (0.19\%) (Fig. 9A). In biopsy samples, the postsynaptic elements of 1276 synapses were determined; $71.63 \%$ of them were AS established on spine heads, $19.36 \%$ were AS on dendritic shafts, $7.29 \%$ were SS on dendritic shafts, and $0.94 \%$ were SS on spine heads (Fig. 9B). The two least frequent types of synapses were AS (0.62\%) and SS (0.16\%) established on spine necks (Fig. 9B).

Regarding the type of synapses (AS or SS), 959 AS and 69 SS were analyzed in the autopsy samples. We found that $68.19 \%$ of the AS were established on spine heads (and $1.36 \%$ on spine necks). The remaining $30.45 \%$ of the AS were established on dendritic shafts $(18.35 \%$ on dendritic shafts with spines and $12.10 \%$ on dendritic shafts without spines). In the case of the SS, $81.16 \%$ of these synapses were established on dendritic shafts (55.07\% on dendritic shafts with spines and $26.09 \%$ on dendritic shafts without spines), while $15.94 \%$ were established on spine heads-and the remaining $2.90 \%$ were found to be on spine necks (Table 5; Supplementary Table 4; Fig. 10).

In the biopsy samples, the postsynaptic elements of 1169 AS and 107 SS were determined. We found that $78.19 \%$ of the AS were established on spine heads and $0.68 \%$ were on spine necks. The remaining $21.13 \%$ of the AS were established on dendritic shafts $(9.15 \%$ on dendritic shafts with spines and $11.98 \%$ on dendritic shafts without spines). Regarding the SS, $86.92 \%$ were established on dendritic shafts $(28.04 \%$ on dendritic shafts with spines and $58.88 \%$ on dendritic shafts without spines), while $11.21 \%$ were established on spine heads-and the remaining $1.87 \%$ were found to be on spine necks (Table 5; Supplementary Table 4; Fig. 10).

Considering all types of synapses established on the spine heads, the proportion of AS:SS was 98:2 and 99:1, in autopsy and biopsy samples, respectively; while in those established on dendritic shafts, this proportion was 84:16 (autopsy) and 
Table 4 Proportion of the different shapes of synaptic junctions in the autopsy and biopsy samples from layer III of BA21

\begin{tabular}{|c|c|c|c|c|c|c|}
\hline Group & $\begin{array}{l}\text { Type of } \\
\text { synapse }\end{array}$ & $\begin{array}{l}\text { Macular } \\
\text { synapses }\end{array}$ & $\begin{array}{l}\text { Perforated } \\
\text { synapses }\end{array}$ & $\begin{array}{l}\text { Horseshoe- } \\
\text { shaped } \\
\text { synapses }\end{array}$ & $\begin{array}{l}\text { Fragmented } \\
\text { synapses }\end{array}$ & $\begin{array}{l}\text { Total } \\
\text { synapses }\end{array}$ \\
\hline \multirow[t]{2}{*}{ Autopsy } & AS & $80.6 \%(1011)$ & $14.5 \%(182)$ & $3.9 \%(49)$ & $1 \%(13)$ & $100 \%(1255)$ \\
\hline & SS & $89 \%(73)$ & $3.7 \%(3)$ & $6.1 \%(5)$ & $1.2 \%(1)$ & $100 \%(82)$ \\
\hline \multirow[t]{2}{*}{ Biopsy } & AS & $84 \%(2826)$ & $11 \%(369)$ & $3.8 \%(127)$ & $1.2 \%(41)$ & $100 \%(3363)$ \\
\hline & SS & $85.3 \%(209)$ & $9.8 \%(24)$ & $4.1 \%(10)$ & $0.8 \%(2)$ & $100 \%(245)$ \\
\hline \multirow[t]{2}{*}{ Total } & AS & $83.1 \%(3837)$ & $11.9 \%(551)$ & $3.8 \%(176)$ & $1.2 \%(54)$ & $100 \%(4618)$ \\
\hline & SS & $86.2 \%(282)$ & $8.3 \%(27)$ & $4.6 \%(15)$ & $0.9 \%(3)$ & $100 \%(327)$ \\
\hline
\end{tabular}

Notes: Data are given as percentages with the absolute number of synapses studied in parentheses. Data for each individual case are shown in Supplementary Table 3. AS: asymmetric synapses; SS: symmetric synapses

Table 5 Distribution of AS and SS on spines and dendritic shafts in autopsy and biopsy samples from layer III of BA21

\begin{tabular}{|c|c|c|c|c|c|c|c|}
\hline Group & $\begin{array}{l}\text { Type of } \\
\text { synapse }\end{array}$ & $\begin{array}{l}\text { Synapses on } \\
\text { spine heads }\end{array}$ & $\begin{array}{l}\text { Synapses on } \\
\text { putative } \\
\text { spine heads }\end{array}$ & $\begin{array}{l}\text { Synapses on } \\
\text { spine necks }\end{array}$ & $\begin{array}{l}\text { Synapses on } \\
\text { dendritic } \\
\text { shaft with } \\
\text { spines }\end{array}$ & $\begin{array}{l}\text { Synapses on } \\
\text { dendritic } \\
\text { shaft } \\
\text { without } \\
\text { spines }\end{array}$ & $\begin{array}{l}\text { Total } \\
\text { synapses }\end{array}$ \\
\hline \multirow[t]{2}{*}{ Autopsy } & AS & $50.57 \%(485)$ & $17.62 \%(169)$ & $1.36 \%(13)$ & $18.35 \%(176)$ & $12.10 \%(116)$ & $100 \%(959)$ \\
\hline & SS & $13.04 \%(9)$ & $2.90 \%(2)$ & $2.90 \%(2)$ & $55.07 \%$ (38) & $26.09 \%(18)$ & $100 \%(69)$ \\
\hline \multirow[t]{2}{*}{ Biopsy } & AS & $56.46 \%(660)$ & $21.73 \%(254)$ & $0.68 \%(8)$ & $9.15 \%(107)$ & $11.98 \%(140)$ & $100 \%(1169)$ \\
\hline & SS & $4.67 \%(5)$ & $6.54 \%(7)$ & $1.87 \%(2)$ & $28.04 \%(30)$ & $58.88 \%(63)$ & $100 \%(107)$ \\
\hline \multirow[t]{2}{*}{ Total } & AS & $53.81 \%(1145)$ & $19.88 \%(423)$ & $0.99 \%(21)$ & $13.30 \%(283)$ & $12.03 \%(256)$ & $100 \%(2128)$ \\
\hline & SS & $7.95 \%$ (14) & $5.11 \%(9)$ & $2.27 \%(4)$ & $38.64 \%(68)$ & $46.02 \%(81)$ & $100 \%$ (176) \\
\hline
\end{tabular}

Notes: Synapses on spines have been subdivided into those established on spine heads that are fully reconstructed or nonfully reconstructed and those established on spine necks. Moreover, we differentiated between dendritic shafts with spines and without spines. Data are given as percentages with the absolute number of synapses studied in parentheses. Data for each individual case are shown in Supplementary Table 4. AS: asymmetric synapses; SS: symmetric synapses

73:27 (biopsy). Since the overall AS:SS ratio was 93:7, the present results show that AS and SS did show a "preference" for a particular postsynaptic element; AS showed a preference for the heads of the spines $\left(\chi^{2}, P<0.0001\right)$, while the SS showed a preference for the dendritic shafts $\left(\chi^{2}, P<0.0001\right.$; Table 5; Fig. 10).

To determine whether there was a difference between the different postsynaptic elements found in the autopsy and the biopsy samples, frequency distribution of the postsynaptic elements, of both AS and SS, were analyzed. Significant differences $\left(\chi^{2}, P<0.0001\right)$ between the autopsy and biopsy samples were found with regard to the AS spine heads and the dendritic shafts frequencies-spine heads were more frequent in the biopsy samples, while dendritic shafts were more frequent in the autopsy samples (Table 5; Fig. 10). Concerning SS, the number of synapses examined was not sufficient to perform a robust statistical analysis.

To detect the presence of multiple synapses, an analysis of the spine heads was performed to determine the number and type of synapses established on them. The most frequent finding was a spine head establishing a single AS $(94.4 \%$ and $96.5 \%$ in the autopsy and biopsy samples, respectively), followed by two AS $(2.7 \%$ and $2.4 \%$ in the autopsy and biopsy samples, respectively), while the proportion of spine heads receiving one AS and one SS was 2.7\% (autopsy samples) and 0.6\% (biopsy samples). The presence of a single SS on a spine head was very low indeed $(0.2 \%$ and $0.5 \%$ in the autopsy and biopsy samples, respectively; Fig. 11). The analysis of the synapses established on spine heads to detect possible differences in the presence of multiple synapses between autopsies and biopsies was discarded due to the low numbers of SS established on spine heads.
Postsynaptic Elements and Synaptic Size

Finally, we analyzed whether there was a relationship between the synaptic size and the type of postsynaptic element. This study was carried out using the data of the area of the SAS of each synapse whose postsynaptic element had been identified as a spine head, spine neck, or a dendritic shaft with or without spines. Synapses established on nonfully reconstructed spines were discarded. AS established on spine heads were larger than AS established on spine necks in both the autopsy (KW; $P=0.0023$ ) and biopsy samples (KW; $P=0.0244$ ). Also, AS established on spine heads were larger than AS established on dendritic shafts in both autopsy (KW, $\mathrm{P}=0.0002)$ and biopsy samples (KW; $P$ < 0.0001) (Supplementary Fig. 6). Concerning SS, the number of synapses was not sufficient to perform a robust statistical analysis.

\section{Discussion}

We have performed a detailed description of the synaptic organization of the neuropil of layer III from BA21 using FIB/SEM in human tissue from both autopsy and biopsy samples. Critical data on synaptic structure and functionality can be obtained from determination of the synaptic density, AS:SS ratio, and 3D spatial distribution, as well as the shape and size of the synapses and their postsynaptic targets. The present results provide a new, large, quantitative ultrastructure dataset in $3 \mathrm{D}$ of the synaptic organization of this particular brain region.

The following major results were obtained: 1) The estimated synaptic density values were lower in autopsy samples than in biopsy samples; 2) the AS:SS ratios were similar in the autopsy 


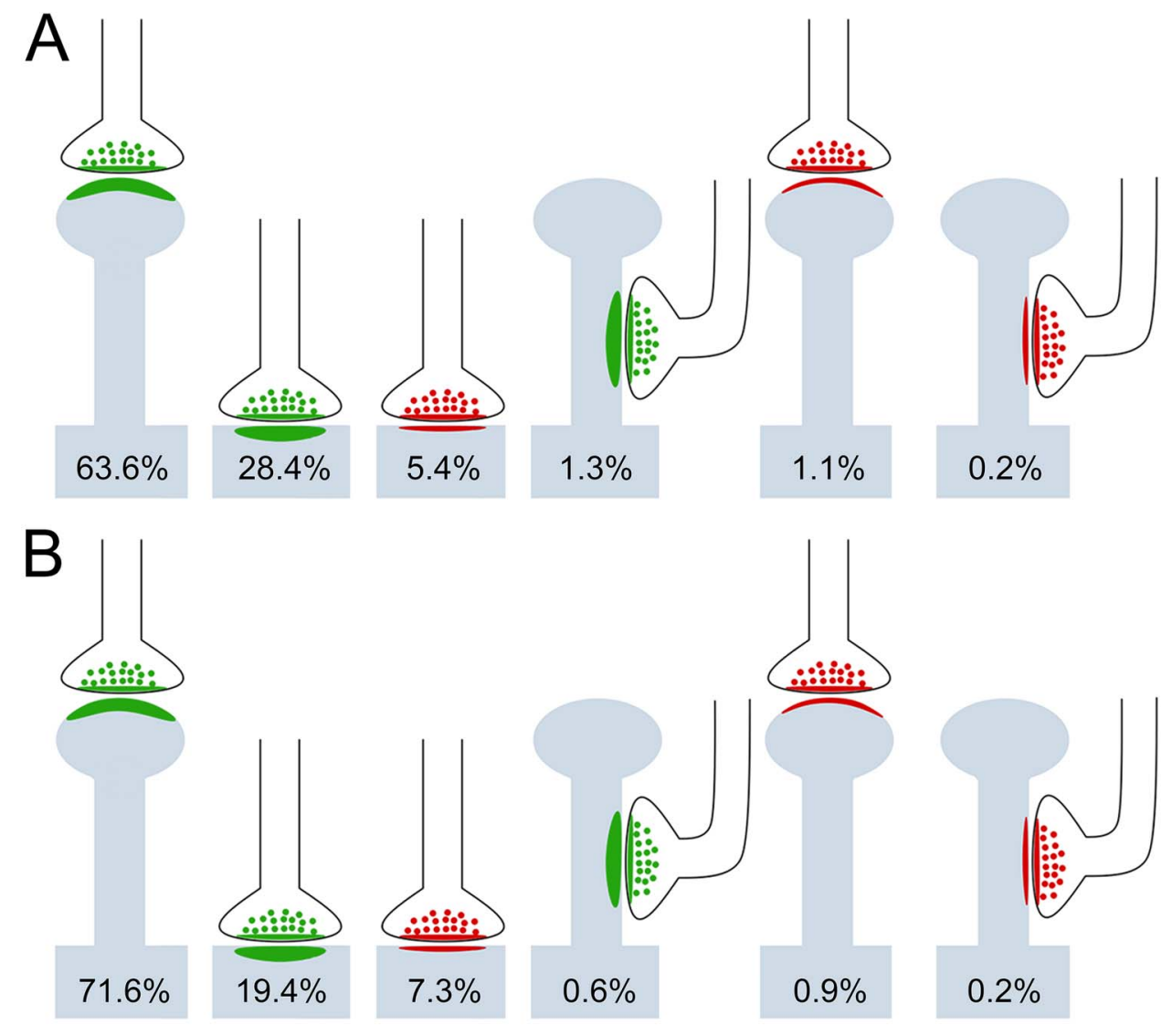

Figure 9. Schematic representation of the distribution of all synapses (AS and SS) on different postsynaptic targets in layer III of BA21. Spine head percentages include both fully reconstructed and nonfully reconstructed spines. Dendritic shaft percentages include dendritic shafts with and without spines. (A) Distribution of synapses on different postsynaptic targets in autopsy samples. (B) Distribution of synapses on different postsynaptic targets in biopsy samples. No significant differences were found between autopsy and biopsy samples. AS have been represented in green and SS in red. AS: asymmetric synapses; SS: symmetric synapses.

and biopsy samples; 3) in all samples, the synapses fitted into a random spatial distribution; 4) the area of the AS was larger than that of SS, in both the autopsy and the biopsy samples; 5) most synapses displayed a macular shape-smaller than complexshaped synapses; 6) most AS were established on dendritic spines, while most SS were established on dendritic shafts; and 7) AS on spine heads were more frequent in the biopsy samples, while dendritic shafts were more frequent in the autopsy samples.

\section{Synaptic Density}

Synaptic density is a critical parameter when it comes to describing the synaptic organization of a particular brain region, in terms of connectivity and functionality. The mean synaptic density found in layer III of the human BA21 was 0.61 synapses $/ \mathrm{\mu m}^{3}$, but differences were found between the autopsy and biopsy samples, with lower values observed in autopsy samples $\left(0.49\right.$ synapses/ $\left.\mu \mathrm{m}^{3}\right)$ than in biopsy samples $(0.66$ synapses $/ \mu^{3}{ }^{3}$. Previous EM studies on the human temporal neocortex have shown either higher synaptic density values in layers II/III (0.73 synapses/ $\mu^{3}$; Davies et al. 1987) and in layer III (0.99 synapses $/ \mathrm{\mu m}^{3}$; Alonso-Nanclares et al. 2008)-or lower values in other layers ( 0.35 and 0.32 synapses $/ \mu^{3}$; Huttenlocher and Dabholkar 1997; Tang et al. 2001; Table 6). A recent study performed in layer IV of the human temporal neocortex reported a synaptic density of 0.002 synapses/ $\mu \mathrm{m}^{3}$ (Yakoubi et al. 2019), which is an extremely low value considering the present results and those reported in the literature (Table 6).

Differences in the estimated synaptic densities (Table 6) may stem from multiple factors, including technical issues affecting the preservation of the ultrastructure. Fixation techniques affect the preservation of the ultrastructure, and therefore the estimated synaptic density may be different from one study to the next. In the present study, although we used the same fixative solution, biopsies were fixed immediately after removal, while tissue from the autopsies was fixed up to $4 \mathrm{~h}$ postmortem. A long postmortem delay for the fixation of brain tissue samples can interfere with synaptic identification (Glausier et al. 2019). Additionally, the dimensions of the brain tissue blocks in biopsies are generally smaller than those from autopsies, which could also influence the penetration of the fixative, such that the fixation in smaller blocks is better (Glausier et al. 2019). The above-mentioned reports from Huttenlocher and Dabholkar (1997) and Tang et al. (2001) used brain tissue samples with a postmortem delay of up to 22 and $60 \mathrm{~h}$, respectively. Therefore, the final values of the synaptic density might be lower due to a lack of proper preservation and identification of the synapses. Moreover, some studies (e.g., Davies et al. 1987; Huttenlocher and Dabholkar 1997) did not take into account that estimations, including morphological measurements, are affected by the tissue shrinkage caused by tissue preparation for electron 


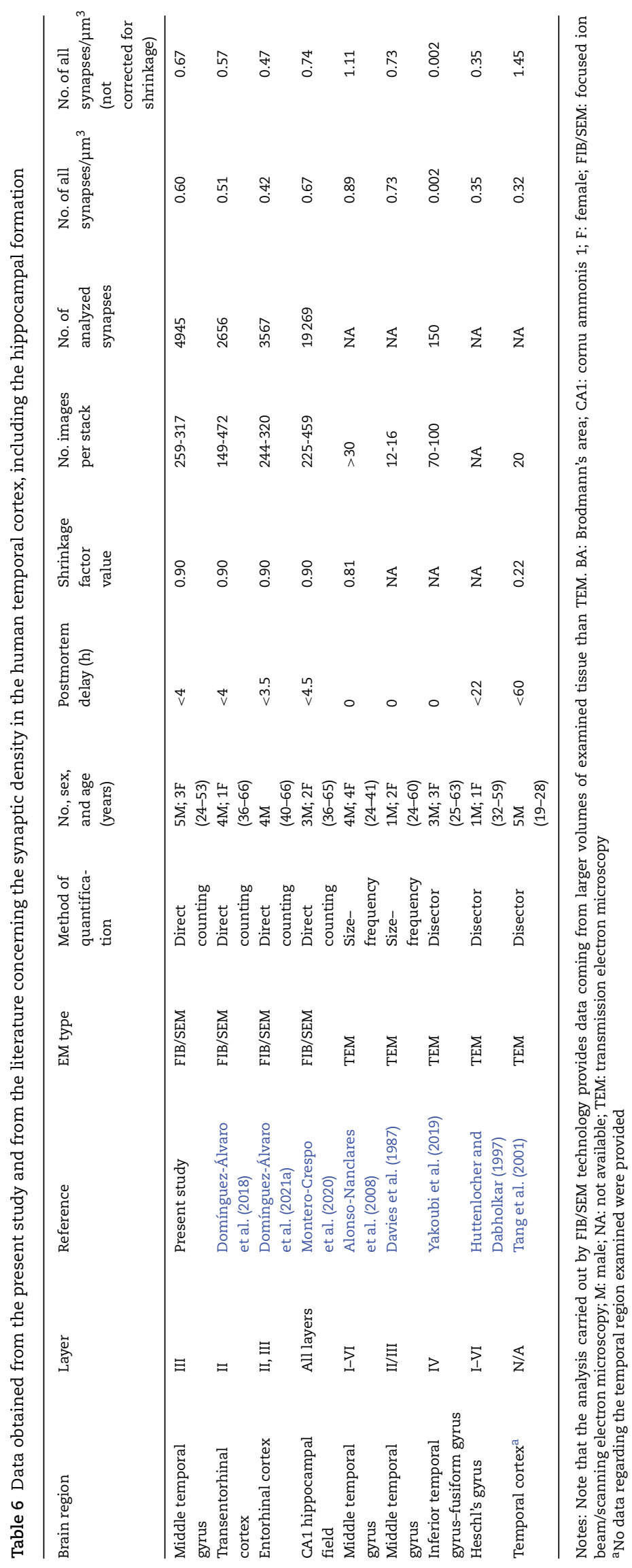




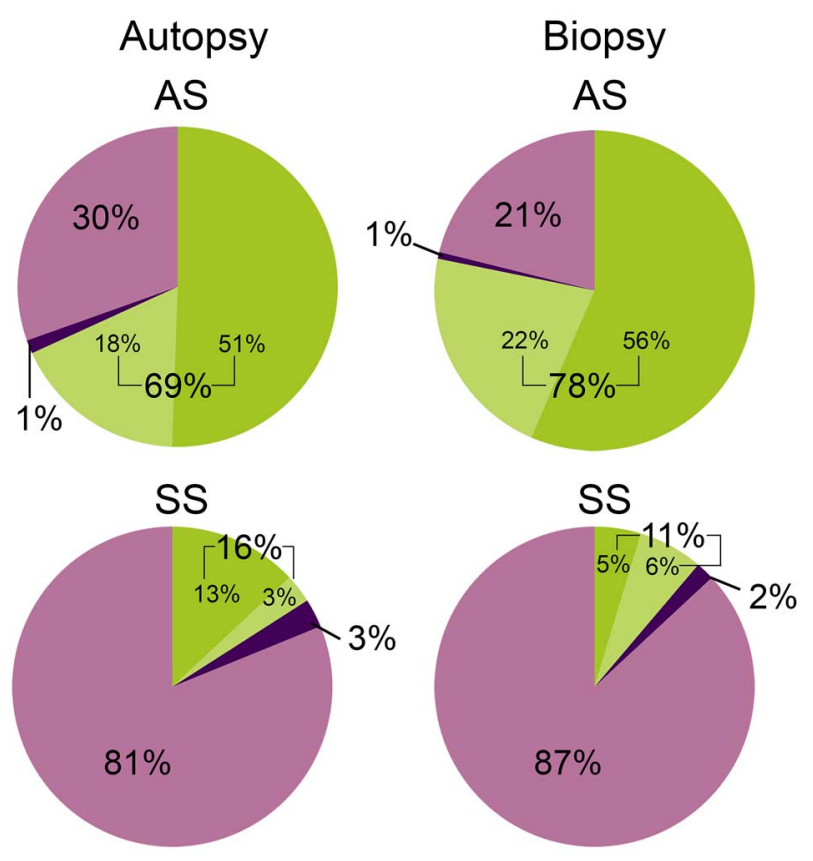

Fully reconstructed spine heads

Non-fully reconstructed spine heads

Spine necks

Dendritic shafts

Figure 10. Proportions of postsynaptic targets of AS and SS in layer III of BA21. In both autopsy and biopsy samples, asymmetric synapses and symmetric synapses show significant preference for spine head targets (including both fully reconstructed and nonfully reconstructed spines) and dendritic shaft targets, respectively $\left(\chi^{2} ; P<0.0001\right)$. Regarding AS, biopsy samples showed a greater proportion of spine head targets than autopsy samples $\left(\chi^{2} ; P<0.0001\right)$. AS: asymmetric synapses; SS: symmetric synapses.

microscopy, which may modify the final estimates. In the present study, we have applied a correction factor to all lineal, surface and volume measurements to overcome the shrinkage derived from electron microscopy processing in chemicalfixed tissue. However, we cannot address how the particular subcellular elements are affected by the chemical fixation. Electron microscopy studies based on cryo-fixed samples seem to be the best option to represent the living state of the tissue. Using this cryo-fixation technique, larger volumes of extracellular space compared with the chemical-fixed samples are reported, and some subcellular elements such as the dendritic spine necks (but not the heads) are shrunken to a greater degree when compared with chemical fixation (Korogod et al. 2015, Tamada et al. 2020). However, as discussed in Tamada et al. (2020), the volumes of well cryo-preserved tissue are still far lower than the volumes that can currently be preserved with chemical fixation, so there are still many limitations with regard to performing a large ultrastructural and quantitative analysis using cryo-fixation techniques. Nevertheless, caution is required when extrapolating the geometrical measurements of ultrastructural elements obtained by chemical fixation to the living state.

Moreover, a lower synapse number has been associated with normal aging in several human neocortical regions (Adams 1987; Benavides-Piccione et al. 2013; Mohan et al. 2018), including the temporal cortex (Berchtold et al. 2013; Henstridge et al. 2016). In our study, the autopsy samples came from individuals aged 45,53 , and 53 years old, whereas the individuals that provided the biopsy samples were younger $(24,27,31,36$, and 41 years old). Therefore, the lower synaptic density observed in autopsy samples $\left(0.49 \pm 0.05 \mathrm{~mm}^{3}\right)$ compared with biopsy samples $\left(0.66 \pm 0.07 \mathrm{~mm}^{3}\right)$ could be related to a combined effect of the fixation techniques and the possible influence of normal aging.

Perhaps more importantly, the differences in the estimated synaptic density reported in the present study compared with that observed in the previous reports may stem from the quantification method and the volume of tissue examined. In some of the above-mentioned TEM studies, synaptic density was calculated using estimations based on the number of synaptic profiles per unit area ("size-frequency method") in single ultrathin sections of tissue, whereas-in others-the "disector method" in several consecutive ultrathin sections was applied. That is, the number of synapses per volume unit was estimated from two-dimensional samples. However, both the sizefrequency and the disector methods are usually applied on a relatively small number of sections due to the difficulties associated with obtaining large numbers of serial sections. As has been shown previously by Merchán-Pérez et al. (2009) and confirmed in the present study, these stereological methods are accurate but only when applied to a large number of sections.

Yakoubi et al. (2019) used the disector method on a large number of excellent-quality TEM serial sections (70-100 sections) from human biopsies belonging to three women and three men, with ages ranging from 25 to 63 years old, with fixation times of $0 \mathrm{~h}$ (Table 6). Thus, their samples might be comparable to ours in terms of ultrastructure preservation and age (Table 1). However, the synaptic density reported by Yakoubi et al. (2019) was more than 100 times lower than our present results and any previously reported values in the human cerebral cortex, including the hippocampal formation (Davies et al. 1987; Huttenlocher and Dabholkar 1997; Tang et al. 2001; Alonso-Nanclares et al. 2008; Montero-Crespo et al. 2020; Domínguez-Álvaro et al. 2021a).

To further examine the influence of the choice of estimation method, we compared the values obtained by direct counting and by the disector method in three stacks of FIB/SEM images. With both methods, we obtained comparable values when all the serial images were used for the estimations $(0.64$ synapses $/ \mu \mathrm{m}^{3}$ by direct counting, and 0.53 synapses $/ \mu \mathrm{m}^{3}$ by the disector method; Supplementary Fig. 3). Indeed, the larger the number of serial sections analyzed, the lower the variability in the final synaptic density estimations. In addition, analysis of 30 TEM images of the same case and layer using the size-frequency method revealed a much higher density (1.04 synapses/ $\mu^{3}$; Alonso-Nanclares et al. 2008). Summing up, a notable difference in the synaptic density values can be observed depending on the method applied and the number of serial sections used.

Previous 3D ultrastructural studies using FIB/SEM in the same autopsy cases as those analyzed in the present study have shown that the synaptic density in other cortical regions-such as layer II of the transentorhinal cortex (0.51 synapses/ $\mathrm{\mu m}^{3}$; Domínguez-Álvaro et al. 2018) and layers II and III of the entorhinal cortex (0.42 synapses $/ \mu^{3}$; Domínguez-Álvaro et al. 2021a)is within the range of the present results. However, estimations in the human hippocampal CA1 field in these autopsy cases have shown that synaptic density ranged from 0.45 to 0.99 synapses $/ \mu^{3}$ (in the stratum oriens and in the superficial 

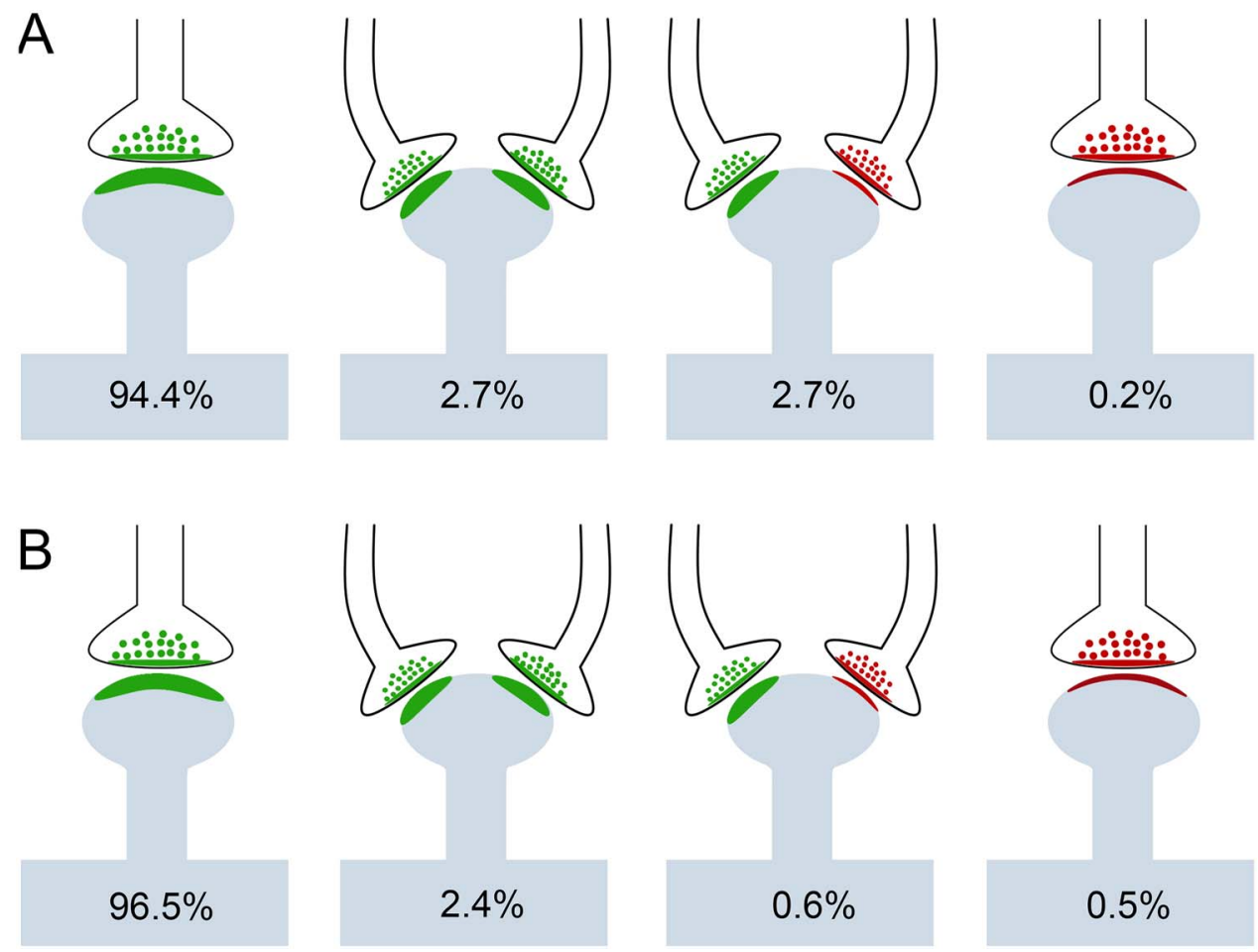

Figure 11. Schematic representation of single and multiple synapses on dendritic spine heads (including both fully reconstructed and nonfully reconstructed spines) in autopsy (A) and biopsy (B) samples. Percentages of each type are indicated. AS have been represented in green and SS in red. AS: asymmetric synapses; SS: symmetric synapses.

part of the stratum pyramidale, respectively) (Montero-Crespo et al. 2020). Since the processing and analysis methods were identical, similarities (to the entorhinal cortices) and differences (with respect to the CA1 superficial pyramidal layer) may be attributable to the specific characteristics of the layer and brain region analyzed, highlighting the fact that synaptic density greatly depends on the human brain layer and/or region being studied (reviewed in DeFelipe 2015).

\section{Proportion of Synapses and Spatial Synaptic Distribution}

It has been established, in different brain regions and species, that the cortical neuropil has a larger proportion of asymmetric synapses than symmetric synapses (reviewed in DeFelipe et al. 2002; DeFelipe 2011). Previous studies have shown that the percentage of AS and SS varies-80-95\% and 20-5\%, respectivelyin all the cortical layers, cortical areas, and species examined so far by TEM (Beaulieu and Colonnier 1985; Megías et al. 2001; Bourne and Harris 2011; DeFelipe 2011, 2015).

In the present study, the AS:SS ratio was 93:7 in both autopsy and biopsy samples, and no differences were found between the two kinds of brain tissue. Similar results have been found using the same methods in the human entorhinal cortex (92:8; Domínguez-Álvaro et al. 2021a), the human transentorhinal cortex (96:4; Domínguez-Álvaro et al. 2018), and the human CA1 hippocampal field (95:5, except in the stratum lacunosum moleculare, in which the AS:SS ratio was 89:11; Montero-Crespo et al. 2020). Thus, it would appear that the present AS:SS ratio data provide among the highest and lowest proportions previously observed in different cortical regions using TEM, for AS and SS, respectively.

The significance of this relatively high proportion of AS and low proportion of SS, and the invariance of this proportion in the human cerebral cortex, are difficult to interpret due to the differences in the cytoarchitecture, connectivity, and functional characteristics of different brain regions.

Since the present results showed similar AS:SS ratios in the neuropil of the above-mentioned brain regions, it could be interpreted that the AS:SS ratio in the dendritic arbor of the different neurons may be similar. However, in other cortical regions of a variety of species, it has been shown that there are differences in the number of inputs of GABAergic and glutamatergic synapses in several neuronal types (e.g., DeFelipe and Fariñas 1992; Freund and Buzsáki 1996; DeFelipe 1997; Somogyi et al. 1998; Schubert et al. 2007; Markram et al. 2015; Tremblay et al. 2016). Thus, it would be necessary to examine the synaptic inputs of each particular cell type to determine possible differences in their AS:SS ratio, even though the overall AS:SS proportion does not vary in the neuropil.

Regarding the spatial organization of synapses, we found that the synapses were randomly distributed in the neuropil samples from both autopsies and biopsies. This type of spatial distribution has also been found in a number of regions of the human brain (frontal cortex, transentorhinal cortex, entorhinal cortex, and CA1 hippocampal field; Blazquez-Llorca et al. 2013; Domínguez-Álvaro et al. 2018, 2021a; Montero-Crespo et al. 2020). Therefore, the present results regarding the random spatial distribution of synapses and the AS:SS proportion (ranging from 92:8 to 96:4) support the idea of widespread "rules" of the synaptic organization of the human cerebral cortex. 


\section{Synaptic Size and Shape}

It has been proposed that synaptic size correlates with release probability, synaptic strength, efficacy, and plasticity (Nusser et al. 1998; Kharazia and Weinberg 1999; Takumi et al. 1999; Ganeshina et al. 2004a; Tarusawa et al. 2009; Matz et al. 2010; Holderith et al. 2012; Südhof 2012; Montes et al. 2015; Biederer et al. 2017). Several methods have traditionally been used to estimate the size of synaptic junctions (Table 7). The simplest of these methods is to measure the cross-sectional length of synaptic junctions in TEM micrographs (Davies et al. 1987; Alonso-Nanclares et al. 2008). This method has obvious limitations since it is based on individual 2D images where a portion of synapses cannot be fully characterized (DeFelipe et al. 1999), and it also reduces size estimation to a 1D measurement. Alternatively, the PSD can be reconstructed from the series of sections and its contour can be measured in 3D (Hsu et al. 2017; Yakoubi et al. 2018, 2019). Another measurement that has been used to estimate the size of synaptic junctions in 3D is the Feret diameter, which is equivalent to the diameter of the smallest sphere circumscribing the reconstructed object (Merchán-Pérez et al. 2014; Calì et al. 2018). However, this does not accurately describe the morphology of synapses, since it obviously oversimplifies the geometric characteristics of the object measured. Another indirect measurement of the size of the synaptic junction is the axon-spine interface, which represents the total apposition surface between the membrane of the axonal bouton and the membrane of the dendritic spine (de Vivo et al. 2017). Although the area of the axon-spine interface and the PSD are correlated (Cheetham et al. 2014), data coming from the axon-spine interface are not comparable with ours, because the SAS is always inside the axon-spine interface and thus it is smaller than the axon-spine interface. Therefore, we have used the SAS, which is equivalent to the interface between the AZ and the PSD. Moreover, our methodology provides information on the shape of the PSD, as well as information about synapses established on dendritic shafts, which cannot be obtained from axon-spine interface measurements.

There are very few studies on human brain that provide data regarding the size of the synaptic junctions. For example, using manual PSD tracing reconstruction in layer IV and V of the temporal neocortex, Yakoubi et al. $(2018,2019)$ reported that the mean size of the PSD was 130000 and $290000 \mathrm{~nm}^{2}$ in layers IV and V, respectively. The values obtained in layer IV were similar to our measurements for the area of the SAS of AS in layer IIIA (110243 $\left.\mathrm{nm}^{2}\right)$, and also similar to our previous results in layer II of the human transentorhinal cortex (118037 nm²; Domínguez-Álvaro et al. 2019) and layers II and III of the entorhinal cortex (110311 and $124183 \mathrm{~nm}^{2}$, respectively; Domínguez-Álvaro et al. 2021a). Thus, it would be necessary to analyze SAS in all cortical layers of BA21 to determine whether layer V has the largest PSD and whether other layers have SAS that are similar to-or smaller than-those found in layers IIIA and IV.

Regarding other species, the synaptic size found in the present study was similar to those described by manual PSD reconstruction in the prefrontal cortex and larger than in the primary visual cortex of the rhesus monkey (110000 and $80000 \mathrm{~nm}^{2}$, respectively; Hsu et al. 2017). The synaptic sizes observed in our results were also larger than those described in rat somatosensory cortex with the same method $\left(65299 \mathrm{~nm}^{2}\right.$; Santuy et al. 2018a). Different synaptic sizes have also been reported in mice with different methods; using manual PSD reconstruction, Hsu et al. (2017) reported a mean synaptic size of 80000 and $70000 \mathrm{~nm}^{2}$ in the primary visual cortex and prefrontal cortex, respectively. Using the Feret diameter, Cali et al. (2018) found a mean synaptic size of $160000 \mathrm{~nm}^{2}$, while using axon-spine interface measurements, de Vivo et al. (2017) reported values of $284000 \mathrm{~nm}^{2}$ in the somatosensory cortex and $294000 \mathrm{~nm}^{2}$ in the primary motor cortex (Table 7).

The present results show that most synapses presented a macular shape (taking both tissue types together, 83\% were macular), whereas $17 \%$ were the more complex-shaped synapses (perforated, horseshoe, and fragmented), which is comparable to previous reports in other brain areas and species (Geinisman et al. 1987; Jones et al. 1991; Neuman et al. 2016; Hsu et al. 2017; Calì et al. 2018; Santuy et al. 2018a; Domínguez-Álvaro et al. 2019; Montero-Crespo et al. 2020).

Complex-shaped synapses are larger than macular ones, and they have more AMPA and NMDA receptors than macular synapses. Therefore, complex synapses are thought to constitute a relatively "powerful" population of synapses with more long-lasting memory-related functionality than macular synapses (Geinisman et al., 1987, 1991, 1992a, 1992b, 1993; Lüscher et al. 2000; Toni et al. 2001; Ganeshina et al. 2004a, 2004b; Spruston 2008). Our present results also showed, in both the autopsy and the biopsy samples, that complex-shaped AS were larger than the macular AS, as was previously reported in other brain areas and species (Santuy et al. 2018a; Domínguez-Álvaro et al. 2019; Montero-Crespo et al. 2020).

In addition, perforated synapses were more frequent in the autopsy samples than in the biopsy samples, and they were also more frequent in AS than in SS. As mentioned above, the autopsy cases were older than the biopsy cases. Thus, the frequency of perforated AS in autopsies could be related to both the fixation process and aging, given that advancing age is associated with a lower number of thin spines (which are the main postsynaptic target of macular AS (Benavides-Piccione et al. 2013).

\section{Postsynaptic Targets}

A clear preference of glutamatergic axons (forming AS) for spines and GABAergic axons (forming SS) for dendritic shafts was observed, which has also been found in a variety of cortical regions and species (reviewed in DeFelipe et al. 2002). In the present study, the co-analysis of the synaptic type and postsynaptic target showed that the proportions of AS established on spines ("axospinous") were around 68\% and $78 \%$ in the autopsy and biopsy samples, respectively. This difference might be explained by the lower number of spines associated with aging (e.g., see Benavides-Piccione et al. 2013) and/or differences in the preservation of the ultrastructure.

Using the same FIB/SEM technology, in layer II of the human transentorhinal cortex, it was found that $75 \%$ of the AS were established on spines (Domínguez-Álvaro et al. 2021b). This percentage was lower for the AS in layers II and III of the human entorhinal cortex-60\% and 56\%, respectively (Domínguez-Álvaro et al. 2021b). These differences and similarities in the proportion of AS on spines and dendritic shafts may represent another microanatomical specialization of the cortical regions (and layers) examined.

Using serial EM, Yakoubi et al. $(2018,2019)$ analyzed 150 AS in layer IV and 147 AS in layer V of the human temporal neocortex, reporting that axospinous AS account for approximately $79 \%$ and $85 \%$ of the AS, respectively (Table 8 ). These results are 


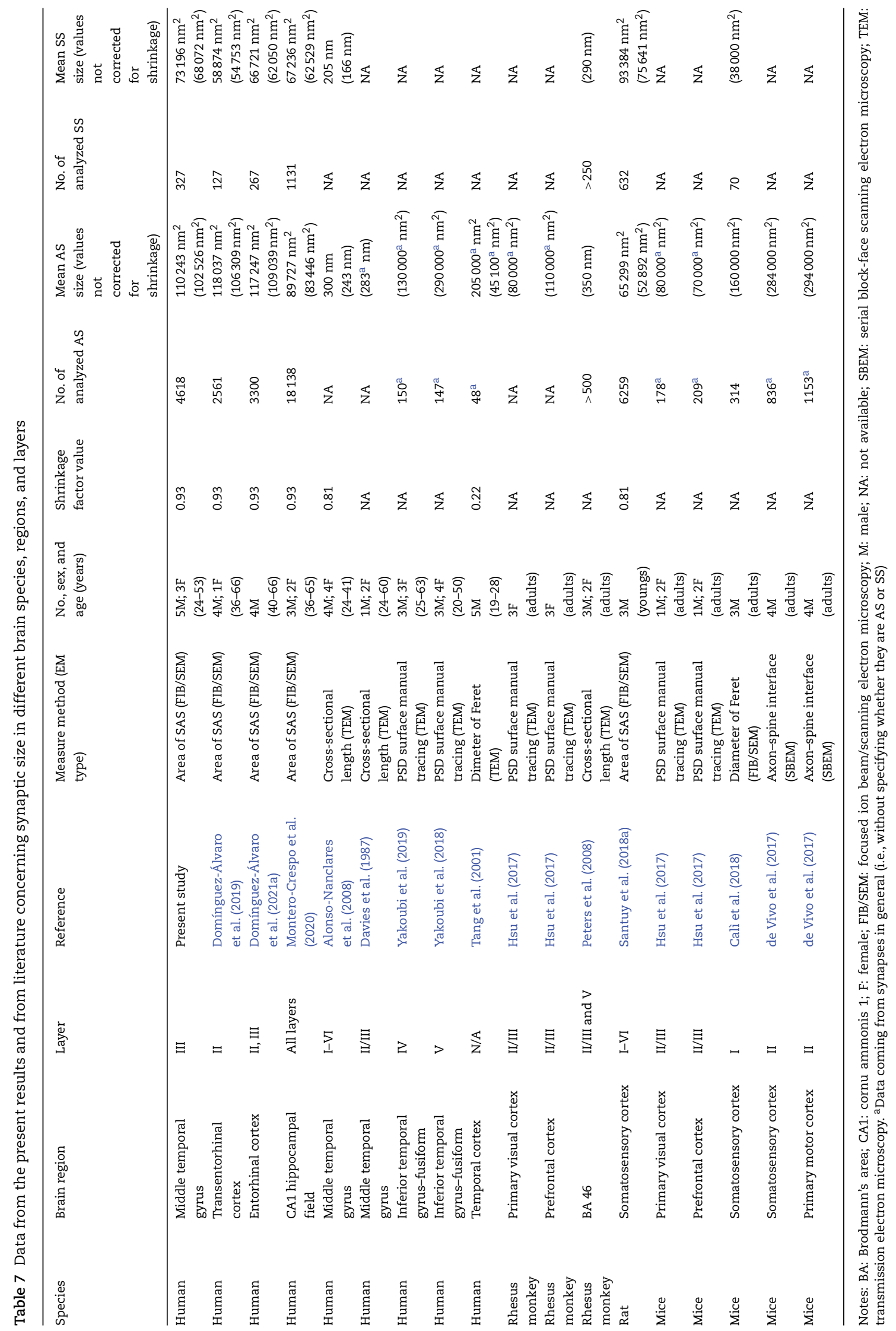




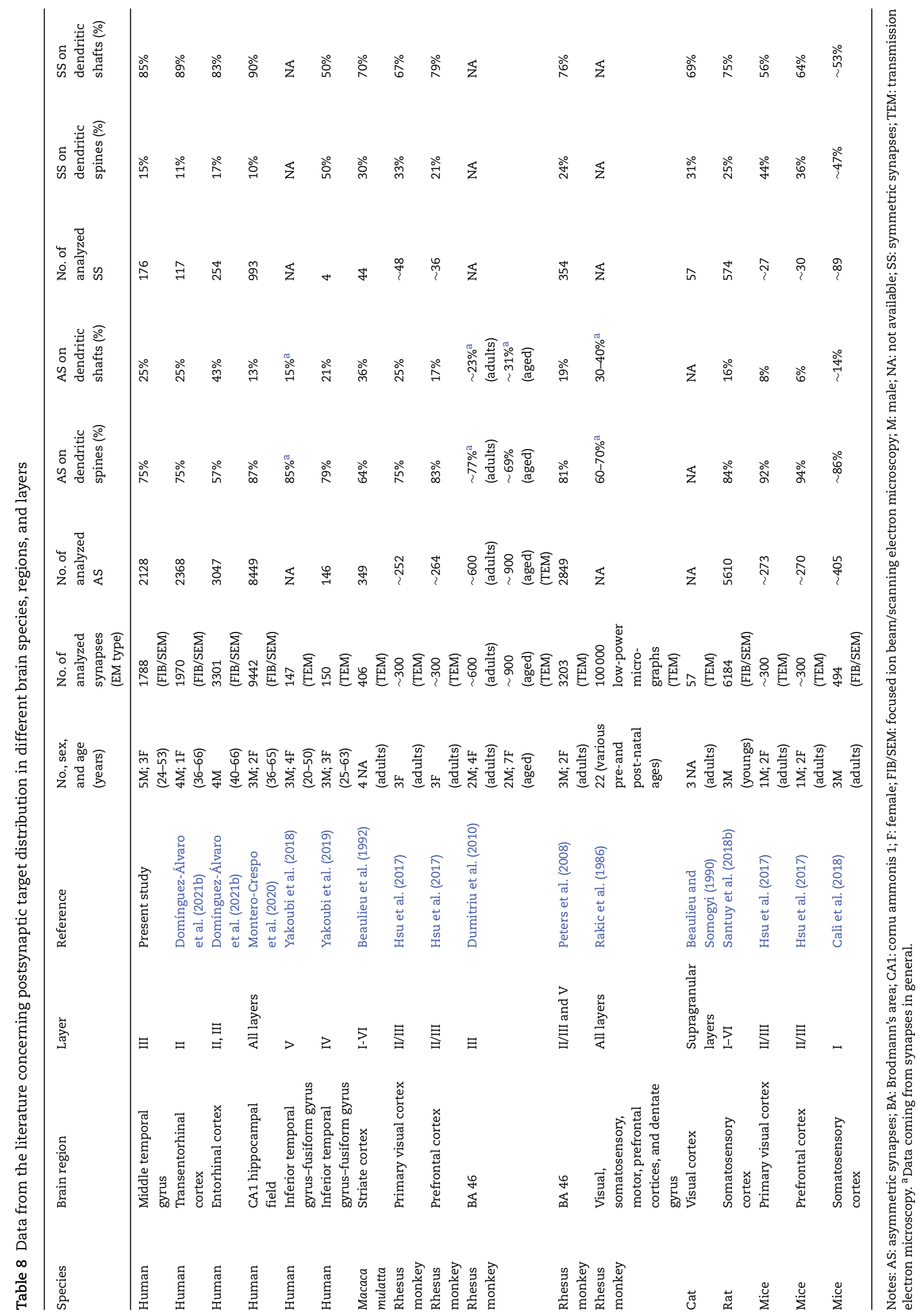


similar to those found in the frontal cortex of the rhesus monkey ( $\sim 80 \%$ axospinous AS; Peters et al. 2008; Dumitriu et al. 2010; Hsu et al. 2017) and higher than those found in the striate cortex of the Macaca mulatta (64\%; Beaulieu et al. 1992). In the present study, we found $70-79 \%$ axospinous AS, from autopsy and biopsy brain tissue, respectively, which is a lower percentage than that found in the study of Yakoubi. Since our results come from the analyses of 2128 AS in layer III, the differences between the findings of Yakoubi et al. and the present results could be related to the number of synapses analyzed (Yakoubi et al. analyzed 150 AS in layer IV and 147 AS in layer VI) and/or to differences in the synaptic organization of the layers examined.

Finally, in rodents, this percentage is higher than in primates-for example, in the somatosensory cortex of the young rat (84\% axospinous AS; Santuy et al. 2018b) and adult mice $(86 \%$ axospinous AS; Calì et al. 2018).

Concerning differences between cortical areas, layers, and species with regard to the targets of SS, it is more difficult to interpret because of the scarcity of SS. As shown in Table 8, most studies are based on analyses of very few SS (4 to 993 synapses). Our data come from 176 serially reconstructed SS and we reported $85 \%$ of SS established on dendritic shafts. Other studies from our laboratory with similar, or even larger, SS datasets have reported similar axodendritic SS percentages in the human transentorhinal cortex (in which $89 \%$ of the 117 SS analyzed were axodendritic SS; Domínguez-Álvaro et al. 2021b) and entorhinal cortex (where $83 \%$ of the 254 SS analyzed were axodendritic SS; Domínguez-Álvaro et al. 2021b). Furthermore, a lower axodendritic SS percentage has been reported in species other than human, including the rhesus monkey (in which $76 \%$ of the 354 SS analyzed [in single sections] in the frontal cortex were axodendritic SS; Peters et al. 2008) and the rat (in which $75 \%$ of the 574 serially reconstructed SS analyzed in the somatosensory cortex were axodendritic; Santuy et al. 2018b). Thus, it may be that GABAergic synapses are organized differently between humans and the other animal species, with human GABAergic circuits being more focused on axodendritic synapses than in the other animal species.

Finally, the present work constitutes a detailed description of the synaptic organization of the neuropil of layer IIIA of the human BA21. Our study is based on the analysis of a large number of synapses (4945 synapses) that were fully reconstructed in $3 \mathrm{D}$ at the ultrastructural level. These results therefore represent robust data for this particular layer. Further studies of the rest of the layers are necessary to further unravel the synaptic organization of this cortical region.

\section{Supplementary Material}

Supplementary Material can be found at Cerebral Cortex online.

\section{Ethics approval}

Brain tissue samples were obtained following the guidelines and approval of the Institutional Ethical Committees from all involved institutions: School of Medicine, University of Castillala Mancha, (Albacete, Spain); and Hospital de la Princesa (Madrid, Spain).

\section{Availability of data and materials}

Most data are available in the main text or the Supplementary Materials. The datasets used and analyzed during the current study are published in the EBRAINS Knowledge Graph (DOI: 10.25493/5F04-N97):

Domínguez-Álvaro M, Montero M, Alonso-Nanclares L, Blazquez-Llorca L, Rodríguez J-R, DeFelipe J. (2020). Densities and 3D distributions of synapses using FIB/SEM imaging in the human neocortex (Temporal cortex, T2) [Data set]. Human Brain Project Neuroinformatics Platform.

\section{Authors' contributions}

J.D. and L.A.-N. designed and oversaw the project. L.A.-N. designed and carried out the experiments. N.C.-A. performed the data analysis. N.C.-A. and L.A.-N. drafted the initial manuscript. All authors read, reviewed, edited, and approved the final manuscript.

\section{Funding}

This work was supported by Spanish "Ministerio de Ciencia e Innovación” (grant PGC2018-094307-B-I00); Cajal Blue Brain Project (the Spanish partner of the Blue Brain Project initiative from EPFL [Switzerland]); Centro de Investigación Biomédica en Red sobre Enfermedades Neurodegenerativas (CIBERNED, Spain, CB06/05/0066); European Union's Horizon 2020 Framework Programme for Research and Innovation (Specific Grant Agreement No. 945539) to Human Brain Project SGA3; and the Spanish "Ministerio de Ciencia e Innovación" (PRE2019-089228 to N.C.-A).

\section{Notes}

We would like to thank Marta Domínguez-Álvaro for her scientific support, Carmen Álvarez and Lorena Valdés for their technical assistance, and Nick Guthrie for his excellent text editing. Conflict of Interest: The authors declare that they have no competing interest.

\section{References}

Adams I. 1987. Comparison of synaptic changes in the precentral and postcentral cerebral cortex of aging humans: a quantitative ultrastructural study. Neurobiol Aging. 8:203-212.

Alonso-Nanclares L, González-Soriano J, Rodríguez J-R, DeFelipe J. 2008. Gender differences in human cortical synaptic density. Proc Natl Acad Sci. 105:14615-14619.

Antón-Sánchez L, Bielza C, Merchán-Pérez A, Rodríguez J-R, DeFelipe J, Larrañaga P. 2014. Three-dimensional distribution of cortical synapses: a replicated point pattern-based analysis. Front Neuroanat. 8:85.

Baddeley A, Rubak E, Turner R. 2015. Spatial point patterns: methodology and applications with R. Boca Raton (CA): Chapman and Hall/CRC Press.

Beaulieu C, Colonnier M. 1985. A laminar analysis of the number of round-asymmetrical and flat-symmetrical synapses on spines, dendritic trunks, and cell bodies in area 17 of the cat. J Comp Neurol. 231:180-189.

Beaulieu C, Somogyi P. 1990. Targets and quantitative distribution of GABAergic synapses in the visual cortex of the cat. Eur J Neurosci. 2:296-303.

Beaulieu C, Kisvarday Z, Somogyi P, Cynader M, Cowey A. 1992. Quantitative distribution of GABA-immunopositive and-immunonegative neurons and synapses in the monkey striate cortex (area 17). Cereb Cortex. 2:295-309. 
Benavides-Piccione R, Fernaud-Espinosa I, Robles V, Yuste R, DeFelipe J. 2013. Age-based comparison of human dendritic spine structure using complete three-dimensional reconstructions. Cereb Cortex. 23:1798-1810.

Berchtold NC, Coleman PD, Cribbs DH, Rogers J, Gillen DL, Cotman CW. 2013. Synaptic genes are extensively downregulated across multiple brain regions in normal human aging and Alzheimer's disease. Neurobiol Aging. 34:1653-1661.

Biederer T, Kaeser PS, Blanpied TA. 2017. Transcellular nanoalignment of synaptic function. Neuron. 96:680-696.

Blazquez-Llorca L, Merchán-Pérez Á, Rodríguez JR, Gascón J, DeFelipe J. 2013. FIB/SEM technology and Alzheimer's disease: three-dimensional analysis of human cortical synapses. J Alzheimers Dis. 34:995-1013.

Bourne JN, Harris KM. 2011. Coordination of size and number of excitatory and inhibitory synapses results in a balanced structural plasticity along mature hippocampal CA1 dendrites during LTP. Hippocampus. 21:354-373.

Calì C, Wawrzyniak M, Becker C, Maco B, Cantoni M, Jorstad A, Nigro B, Grillo F, De Paola V, Fua P et al. 2018. The effects of aging on neuropil structure in mouse somatosensory cortex-a 3D electron microscopy analysis of layer 1. PLoS One. 13:e0198131.

Cheetham CE, Barnes SJ, Albieri G, Knott GW, Finnerty GT. 2014. Pansynaptic enlargement at adult cortical connections strengthened by experience. Cereb Cortex. 24:521-531.

Cheng W, Rolls ET, Zhang J, Sheng W, Ma L, Wan L, Luo Q, Feng J. 2017. Functional connectivity decreases in autism in emotion, self, and face circuits identified by knowledge-based enrichment analysis. Neuroimage. 148:169-178.

Davies CA, Mann DMA, Sumpter PQ, Yates PO. 1987. A quantitative morphometric analysis of the neuronal and synaptic content of the frontal and temporal cortex in patients with Alzheimer's disease. J Neurol Sci. 78:151-164.

DeFelipe J. 1997. Types of neurons, synaptic connections and chemical characteristics of cells immunoreactive for calbindin-D28K, parvalbumin and calretinin in the neocortex. J Chem Neuroanat. 14:1-19.

DeFelipe J. 2011. The evolution of the brain, the human nature of cortical circuits, and intellectual creativity. Front Neuroanat. 5.

DeFelipe J. 2015. The anatomical problem posed by brain complexity and size: a potential solution. Front Neuroanat. 9.

DeFelipe J, Fairén A. 1993. A simple and reliable method for correlative light and electron microscopic studies.J Histochem Cytochem. 41:769-772.

DeFelipe J, Fariñas I. 1992. The pyramidal neuron of the cerebral cortex: morphological and chemical characteristics of the synaptic inputs. Prog Neurobiol. 39:563-607.

DeFelipe J, Marco P, Busturia I, Merchán-Pérez A. 1999. Estimation of the number of synapses in the cerebral cortex: methodological considerations. Cereb Cortex. 9:722-732.

DeFelipe J, Alonso-Nanclares L, Arellano J. 2002. Microstructure of the neocortex: comparative aspects. J Neurocytol. 31:299-316.

del Río MR, DeFelipe J. 1994. A study of SMI 32-stained pyramidal cells, parvalbumin-immunoreactive chandelier cells, and presumptive thalamocortical axons in the human temporal neocortex: pyramidal cells, chandelier cells, and thalamocortical axons. J Comp Neurol. 342:389-408.

del Río MR, DeFelipe J. 1995. A light and electron microscopic study of Calbindin D-28k immunoreactive double bouquet cells in the human temporal cortex. Brain Res. 690:133-140.
Denk W, Horstmann H. 2004. Serial block-face scanning electron microscopy to reconstruct three-dimensional tissue nanostructure. PLoS Biol. 2:e329.

de Vivo L, Bellesi M, Marshall W, Bushong EA, Ellisman MH, Tononi G, Cirelli C. 2017. Ultrastructural evidence for synaptic scaling across the wake/sleep cycle. Science. 355(6324):507-510.

Domínguez-Álvaro M, Montero-Crespo M, Blazquez-Llorca L, Insausti R, DeFelipe J, Alonso-Nanclares L. 2018. Threedimensional analysis of synapses in the transentorhinal cortex of Alzheimer's disease patients. Acta Neuropathol Commun. 6:20.

Domínguez-Álvaro M, Montero-Crespo M, Blazquez-Llorca L, DeFelipe J, Alonso-Nanclares L. 2019. 3D electron microscopy study of Synaptic Organization of the Normal Human Transentorhinal Cortex and its possible alterations in Alzheimer's disease. eNeuro. 6:ENEURO.0140-19.2019.

Domínguez-Álvaro M, Montero-Crespo M, Blazquez-Llorca L, DeFelipe J, Alonso-Nanclares L. 2021a. 3D ultrastructural study of synapses in the human entorhinal. Cereb Cortex. 31:410-425.

Domínguez-Álvaro M, Montero-Crespo M, Blazquez-Llorca L, Plaza-Alonso S, Cano-Astorga N, DeFelipe J, AlonsoNanclares L. 2021b. 3D analyses of the synaptic organization in the entorhinal cortex in Alzheimer's disease. eNeuro. In press.

Dumitriu D, Hao J, Hara Y, Kaufmann J, Janssen WGM, Lou W, Rapp PR, Morrison JH. 2010. Selective changes in thin spine density and morphology in monkey prefrontal cortex correlate with aging-related cognitive impairment. J Neurosci. 30:7507-7515.

Eyal G, Verhoog MB, Testa-Silva G, Deitcher Y, BenavidesPiccione R, DeFelipe J, de Kock CPJ, Mansvelder HD, Segev I. 2018. Human cortical pyramidal neurons: from spines to spikes via models. Front Cell Neurosci. 12:181.

Freund TF, Buzsáki G. 1996. Interneurons of the hippocampus. Hippocampus. 6:347-470.

Froemke RC. 2015. Plasticity of cortical excitatory-inhibitory balance. Annu Rev Neurosci. 38:195-219.

Ganeshina O, Berry RW, Petralia RS, Nicholson DA, Geinisman Y. 2004a. Differences in the expression of AMPA and NMDA receptors between axospinous perforated and nonperforated synapses are related to the configuration and size of postsynaptic densities. J Comp Neurol. 468:86-95.

Ganeshina O, Berry RW, Petralia RS, Nicholson DA, Geinisman Y. 2004b. Synapses with a segmented, completely partitioned postsynaptic density express more AMPA receptors than other axospinous synaptic junctions. Neuroscience. 125:615-623.

Geinisman Y, Morrell F, de Toledo-Morrell L. 1987. Axospinous synapses with segmented postsynaptic densities: a morphologically distinct synaptic subtype contributing to the number of profiles of 'perforated' synapses visualized in random sections. Brain Res. 423:179-188.

Geinisman Y, Morrell F, de Toledo-Morrell L. 1991. Induction of long-term potentiation is associated with an increase in the number of axospinous synapses with segmented postsynaptic densities. Brain Res. 566:77-88.

Geinisman Y, de Toledo-Morrell L, Morrell F, Persina IS, Rossi M. 1992a. Structural synaptic plasticity associated with the induction of long-term potentiation is preserved in the dentate gyrus of aged rats. Hippocampus. 2:445-456. 
Geinisman Y, Morrell F, deToledo-Morrell L. 1992b. Increase in the number of axospinous synapses with segmented postsynaptic densities following hippocampal kindling. Brain Res. 569:341-347.

Geinisman Y, de Toledo-Morrell L, Morrell F, Heller RE, Rossi M, Parshall RF. 1993. Structural synaptic correlate of long-term potentiation: formation of axospinous synapses with multiple, completely partitioned transmission zones. Hippocampus. 3:435-445.

Gidon A, Zolnik TA, Fidzinski P, Bolduan F, Papoutsi A, Poirazi P, Holtkamp M, Vida I, Larkum ME. 2020. Dendritic action potentials and computation in human layer $2 / 3$ cortical neurons. Science. 367:83-87.

Glausier JR, Konanur A, Lewis DA. 2019. Factors affecting ultrastructural quality in the prefrontal cortex of the postmortem human brain. J Histochem Cytochem. 67:185-202.

Gray EG. 1959. Axo-somatic and axo-dendritic synapses of the cerebral cortex: an electron microscope study. J Anat. 4:420-433.

Gundersen HJG. 1977. Notes on the estimation of the numerical density of arbitrary profiles: the edge effect. J Microsc. 111:219-223.

Gundersen HJG, Bagger P, Bendtsen TF, Evans SM, Korbo L, Marcussen N, MØLler A, Nielsen K, Nyengaard JR, Pakkenberg B et al. 1988a. The new stereological tools: disector, fractionator, nucleator and point sampled intercepts and their use in pathological research and diagnosis. APMIS. 96:857-881.

Gundersen HJG, Bendtsen TF, Korbo L, Marcussen N, Møller A, Nielsen K, Nyengaard JR, Pakkenberg B, Sørensen FB, Vesterby A et al. 1988b. Some new, simple and efficient stereological methods and their use in pathological research and diagnosis. APMIS. 96:379-394.

Helmstaedter M, Briggman KL, Turaga SC, Jain V, Seung HS, Denk W. 2013. Connectomic reconstruction of the inner plexiform layer in the mouse retina. Nature. 500:168-174.

Henstridge CM, Pickett E, Spires-Jones TL. 2016. Synaptic pathology: a shared mechanism in neurological disease. Aging Res Rev. 28:72-84.

Holderith N, Lorincz A, Katona G, Rózsa B, Kulik A, Watanabe M, Nusser Z. 2012. Release probability of hippocampal glutamatergic terminals scales with the size of the active zone. Nat Neurosci. 15:988-997.

Hsu A, Luebke JI, Medalla M. 2017. Comparative ultrastructural features of excitatory synapses in the visual and frontal cortices of the adult mouse and monkey. J Comp Neurol. 525:2175-2191.

Huttenlocher PR, Dabholkar AS. 1997. Regional differences in synaptogenesis in human cerebral cortex. J Comp Neurol. 387:167-178.

Jones DG, Itarat W, Calverley RKS. 1991. Perforated synapses and plasticity: a developmental overview. Mol Neurobiol. 5:217-228.

Kasthuri N, Hayworth KJ, Berger DR, Schalek RL, Conchello JA, Knowles-Barley S, Lee D, Vázquez-Reina A, Kaynig V, Jones TR et al. 2015. Saturated reconstruction of a volume of neocortex. Cell. 162:648-661.

Kharazia VN, Weinberg RJ. 1999. Immunogold localization of AMPA and NMDA receptors in somatic sensory cortex of albino rat. J Comp Neurol. 412:292-302.

Kleinfeld D, Bharioke A, Blinder P, Bock DD, Briggman KL, Chklovskii DB, Denk W, Helmstaedter M, Kaufhold JP, Lee WCA et al. 2011. Large-scale automated histology in the pursuit of connectomes. J Neurosci. 31:16125-16138.
Knott G, Marchman H, Wall D, Lich B. 2008. Serial section scanning electron microscopy of adult brain tissue using focused ion beam milling. J Neurosci. 28:2959-2964.

Korogod N, Petersen CC, Knott GW. 2015. Ultrastructural analysis of adult mouse neocortex comparing aldehyde perfusion with cryo fixation. Elife. 4:e05793.

Kubota Y, Sohn J, Kawaguchi Y. 2018. Large volume electron microscopy and neural microcircuit analysis. Front Neural Circuits. 12:98.

Lüscher C, Nicoll RA, Malenka RC, Muller D. 2000. Synaptic plasticity and dynamic modulation of the postsynaptic membrane. Nat Neurosci. 3:545-550.

Markram H, Muller E, Ramaswamy S, Reimann MW, Abdellah M, Sanchez CA, Ailamaki A, Alonso-Nanclares L, Antille N, Arsever $S$ et al. 2015. Reconstruction and simulation of neocortical microcircuitry. Cell. 163:456-492.

Matz J, Gilyan A, Kolar A, McCarvill T, Krueger SR. 2010. Rapid structural alterations of the active zone lead to sustained changes in neurotransmitter release. Proc Natl Acad Sci. 107:8836-8841.

Megías M, Emri Z, Freund TF, Gulyás AI. 2001. Total number and distribution of inhibitory and excitatory synapses on hippocampal CA1 pyramidal cells. Neuroscience. 102:527-540.

Merchán-Pérez A, Rodríguez J-R, Alonso-Nanclares L, Schertel A, DeFelipe J. 2009. Counting synapses using FIB/SEM microscopy: a true revolution for ultrastructural volume reconstruction. Front Neuroanat. 3:18.

Merchán-Pérez A, Rodríguez J-R, González S, Robles V, DeFelipe J, Larrañaga P, Bielza C. 2014. Three-dimensional spatial distribution of synapses in the neocortex: a dual-beam electron microscopy study. Cereb Cortex. 24:1579-1588.

Mohan A, Thalamuthu A, Mather KA, Zhang Y, Catts VS, Weickert CS, Sachdev PS. 2018. Differential expression of synaptic and interneuron genes in the aging human prefrontal cortex. Neurobiol Aging. 70:194-202.

Montero-Crespo M, Domínguez-Álvaro M, Rondón-Carrillo P, Alonso-Nanclares L, DeFelipe J, Blazquez-Llorca L. 2020. Three-dimensional synaptic organization of the human hippocampal CA1 field. Elife. 9:e57013.

Montes J, Peña JM, DeFelipe J, Herreras O, Merchan-Perez A. 2015. The influence of synaptic size on AMPA receptor activation: a Monte Carlo model. PLoS One. 10:e0130924.

Morales J, Rodríguez A, Rodríguez J-R, DeFelipe J, MerchánPérez A. 2013. Characterization and extraction of the synaptic apposition surface for synaptic geometry analysis. Front Neuroanat. 7:20.

Neuman KM, Molina-Campos E, Musial TF, Price AL, Oh K-J, Wolke ML, Buss EW, Scheff SW, Mufson EJ, Nicholson DA. 2016. Evidence for Alzheimer's disease-linked synapse loss and compensation in mouse and human hippocampal CA1 pyramidal neurons. Brain Struct Funct. 220:3143-3165.

Nusser Z, Hájos N, Somogyi P, Mody I. 1998. Increased number of synaptic GABAA receptors underlies potentiation at hippocampal inhibitory synapses. Nature. 395:172-177.

Oorschot D, Peterson D, Jones D. 1991. Neurite growth from, and neuronal survival within, cultured explants of the nervous system: a critical review of morphometric and stereological methods, and suggestions for the future. Prog Neurobiol. 37:525-546.

Peters A, Palay SL, Webster HD. 1991. The fine structure of the nervous system: The neurons and their supporting cells. New York: Oxford University. 
Peters A, Sethares C, Luebke JI. 2008. Synapses are lost during aging in the primate prefrontal cortex. Neuroscience. 152:970-981.

Rakic P, Bourgeois J, Eckenhoff M, Zecevic N, GoldmanRakic P. 1986. Concurrent overproduction of synapses in diverse regions of the primate cerebral cortex. Science. 232:232-235.

Santuy A, Rodríguez J-R, DeFelipe J, Merchán-Pérez A. 2018a. Study of the size and shape of synapses in the juvenile rat somatosensory cortex with 3D electron microscopy. eNeuro. 5:ENEURO.0377-17.2017.

Santuy A, Rodríguez J-R, DeFelipe J, Merchán-Pérez A. 2018b. Volume electron microscopy of the distribution of synapses in the neuropil of the juvenile rat somatosensory cortex. Brain Struct Funct. 223:77-90.

Schubert D, Kötter R, Staiger JF. 2007. Mapping functional connectivity in barrel-related columns reveals layer- and cell type-specific microcircuits. Brain Struct Funct. 212:107-119.

Smith DJ. 2008. Progress and perspectives for atomic-resolution electron microscopy. Ultramicroscopy. 108:159-166.

Sohal VS, Rubenstein JLR. 2019. Excitation-inhibition balance as a framework for investigating mechanisms in neuropsychiatric disorders. Mol Psychiatry. 24:1248-1257.

Somogyi P, Tamas G, Lujan R, Buhl EH. 1998. Salient features of synaptic organisation in the cerebral cortex. Brain Res Rev. 26:113-135.

Spruston N. 2008. Pyramidal neurons: dendritic structure and synaptic integration. Nat Rev Neurosci. 9:206-221.

Südhof TC. 2012. The presynaptic active zone. Neuron. 75:11-25.

Takumi Y, Ramírez-León V, Laake P, Rinvik E, Ottersen OP. 1999. Different modes of expression of AMPA and NMDA receptors in hippocampal synapses. Nat Neurosci. 2:618-624.

Tamada H, Blanc J, Korogod N, Petersen CC, Knott GW. 2020. Ultrastructural comparison of dendritic spine morphology preserved with cryo and chemical fixation. Elife. 9:e56384.

Tang Y, Nyengaard JR, De Groot DMG, Gundersen HJG. 2001. Total regional and global number of synapses in the human brain neocortex. Synapse. 41:258-273.

Tarusawa E, Matsui K, Budisantoso T, Molnar E, Watanabe M, Matsui M, Fukazawa Y, Shigemoto R. 2009. Input-specific Intrasynaptic arrangements of ionotropic glutamate receptors and their impact on postsynaptic responses. J Neurosci. 29:12896-12908.

Thomson AM, Lamy C. 2007. Functional maps of neocortical local circuitry. Front Neurosci. 1:19-42.

Toni N, Buchs P-A, Nikonenko I, Povilaitite P, Parisi L, Muller D. 2001. Remodeling of synaptic membranes after induction of long-term potentiation. J Neurosci. 21:6245-6251.

Tremblay R, Lee S, Rudy B. 2016. GABAergic interneurons in the neocortex: from cellular properties to circuits. Neuron. 91:260-292.

Xu Y, Lin Q, Han Z, He Y, Bi Y. 2016. Intrinsic functional network architecture of human semantic processing: modules and hubs. Neuroimage. 132:542-555.

Yakoubi R, Rollenhagen A, von Lehe M, Shao Y, Sätzler K, Lübke JHR. 2018. Quantitative three-dimensional reconstructions of excitatory synaptic boutons in layer 5 of the adult human temporal lobe neocortex: a fine-scale electron microscopic analysis. Cereb Cortex. 29:2797-2814.

Yakoubi R, Rollenhagen A, von Lehe M, Miller D, Walkenfort B, Hasenberg M, Sätzler K, Lübke JH. 2019. Ultrastructural heterogeneity of layer 4 excitatory synaptic boutons in the adult human temporal lobe neocortex. Elife. 8:e48373.

Zhou S, Yu Y. 2018. Synaptic E-I balance underlies efficient neural coding. Front Neurosci. 12:46.

Zilles K, Amunts K. 2010. Centenary of Brodmann's mapconception and fate. Nat Rev Neurosci. 11:139-145. 